\title{
Catalog of Differentially Expressed Long Non-Coding RNA following Activation of Human and Mouse Innate Immune Response
}

\author{
Benoit T. Roux 1 , James A. Heward ${ }^{2}$, Louise E. Donnelly ${ }^{3}$, Simon W. Jones ${ }^{4}$ \\ and Mark A. Lindsay ${ }^{1,3 *}$
}

'Department of Pharmacy and Pharmacology, University of Bath, Bath, United Kingdom, ${ }^{2}$ Barts Cancer Institute, Queen Mary University of London, London, United Kingdom, ${ }^{3}$ Airway Disease, National Heart and Lung Institute, Imperial College, London, United Kingdom, ${ }^{4}$ Institute of Inflammation and Ageing, MRC-ARUK Centre for Musculoskeletal Ageing Research, University of Birmingham, Birmingham, United Kingdom

OPEN ACCESS

Edited by:

Olivier Neyrolles,

Centre national de la recherche scientifique (CNRS), France

Reviewed by: Mattia Pelizzola,

Fondazione Istituto Italiano di

Technologia, Italy

Camille Lobry,

Institut Gustave Roussy, France

*Correspondence:

Mark A. Lindsay

m.a.lindsay@bath.ac.uk

Specialty section:

This article was submitted to

Molecular Innate Immunity,

a section of the journal

Frontiers in Immunology

Received: 06 June 2017 Accepted: 11 August 2017

Published: 29 August 2017

Citation:

Roux BT, Heward JA, Donnelly LE, Jones SW and Lindsay MA (2017) Catalog of Differentially Expressed Long Non-Coding RNA following Activation of Human and Mouse Innate Immune Response.

Front. Immunol. 8:1038. doi: 10.3389/fimmu.2017.01038
Despite increasing evidence to indicate that long non-coding RNAs (IncRNAs) are novel regulators of immunity, there has been no systematic attempt to identify and characterize the IncRNAs whose expression is changed following the induction of the innate immune response. To address this issue, we have employed next-generation sequencing data to determine the changes in the IncRNA profile in four human (monocytes, macrophages, epithelium, and chondrocytes) and four mouse cell types (RAW 264.7 macrophages, bone marrow-derived macrophages, peritoneal macrophages, and splenic dendritic cells) following exposure to the pro-inflammatory mediators, lipopolysaccharides (LPS), or interleukin-1 $\beta$. We show differential expression of 204 human and 210 mouse IncRNAs, with positional analysis demonstrating correlation with immune-related genes. These IncRNAs are predominantly cell-type specific, composed of large regions of repeat sequences, and show poor evolutionary conservation. Comparison within the human and mouse sequences showed less than $1 \%$ sequence conservation, although we identified multiple conserved motifs. Of the 204 human IncRNAs, 21 overlapped with syntenic mouse IncRNAs, of which five were differentially expressed in both species. Among these syntenic IncRNA was IL7-AS (antisense), which was induced in multiple cell types and shown to regulate the production of the pro-inflammatory mediator interleukin- 6 in both human and mouse cells. In summary, we have identified and characterized those IncRNAs that are differentially expressed following activation of the human and mouse innate immune responses and believe that these catalogs will provide the foundation for the future analysis of the role of IncRNAs in immune and inflammatory responses.

Keywords: long non-coding RNA, innate immunity, conserved motif, human catalog, mouse catalog, inflammation

Abbreviations: BMDM, bone marrow-derived macrophages; mRNA, messenger RNA; ncRNA, non-coding RNA; IncRNA, long non-coding RNA; lincRNA, long intergenic non-coding RNA; IL, interleukin; LPS, lipopolysaccharide; nt, nucleotide. 


\section{INTRODUCTION}

High-throughput sequencing indicates that much of the human genome is transcribed into non-coding RNAs (ncRNAs) with estimates of the proportion varying from $\sim 62 \%$ predicted by the ENCODE project (1) to $\sim 10 \%$ based on evolutionary conservation (2). By absolute amount, the majority of ncRNAs ( $>90 \%)$ are involved in house-keeping activities such as translation, splicing, and post-transcriptional RNA modifications and include ribosomal RNAs, transfer RNAs, short nucleolar RNAs, and small nuclear RNAs $(3,4)$. The remaining ncRNAs are broadly classified as either short ncRNAs [ $<200$ nucleotides (nt)] or long ncRNAs (lncRNAs) (>200 nt) (4). The microRNA family of short ncRNAs is the best characterized and is known to induce messenger RNA (mRNA) degradation or block mRNA translation via the RNA interference pathway (5). By contrast, much less in known about lncRNAs, although, by comparison with mRNAs, their expression is cell specific and they are generally shorter in length, contain fewer exons, and are expressed at lower levels $(6,7)$. Presently, lncRNAs are classified by their relative position to protein-coding mRNAs and include the long intergenic ncRNAs (lincRNAs), antisense (AS), and pseudogenes (8). Although there is accumulating evidence showing that lncRNAs are regulators of a host of physiological and pathological responses, our understanding of their mechanism of action is limited. By analogy with protein-coding genes, it has been speculated that this is mediated through domains that interact with proteins and/or base pair with RNA/DNA (9). However, the identification of these domains has been hindered by their poor evolutionary conservation, which, in contrast to protein-coding genes, does not require the maintenance of a conserved open reading frame for optimal translation (6). Instead, it is thought that the IncRNAs conservation is geared toward the maintenance of genomic position (synteny), short domains (microdomains), and secondary structure $(7,10)$.

The innate immune response provides the initial defense against infection by external pathogens through induction of an inflammatory response. The presence of pathogens is commonly detected by cells of the myeloid family, including tissue resident macrophages, dendritic cells, and circulating blood monocytes $(11,12)$. These cells express families of pattern recognition receptors that bind conserved molecules within bacteria, fungi, and viruses including lipoproteins, lipopolysaccharides (LPS), bacterial CpG motifs, and single/double-stranded RNA. Many families of pattern recognition receptors have been identified, although the best characterized are the toll-like receptor and interleukin-1 $\beta$ (IL1 $\beta$ ) receptor superfamily. Activation of Toll-like receptors stimulates the production of inflammatory mediators via transcription factors including nuclear factor- $\mathrm{KB}$ (NF- $\mathrm{KB}$ ). This leads to a spectrum of responses including the release of multiple inflammatory mediators, as well as the activation of the inflammasome and the subsequent production of IL1 $\beta$. The latter then induces a potent inflammatory response in the surrounding stromal cells such as the epithelium, chondrocytes, and fibroblasts $(11,12)$.

Recent publications have identified a number of lncRNAs that are differentially expressed following activation of innate immunity and which regulate the subsequent inflammatory response. In human cells, these include PACER (p50-associated COX-2 extragenic RNA) (13), THRIL (TNF $\alpha$ - and hnRNPLrelated immunoregulatory lincRNA) (14), $\ln c-I L 7 R$ (15), and IL1 $\beta$-RBT46 (16), while studies in mice have identified lincRNACOX2 (17, 18), lincRNA-EPS (19), and lincRNA-Tnfaip3 (20). However, despite these early indications that lncRNAs act as novel regulators, there has been no systematic attempt to identify lncRNAs whose expression is changed following the induction of the innate immune response. To address this issue, we determined the changes in lncRNA profile in four human and four mouse cell types following exposure to LPS or IL1 $\beta$. From this analysis, we have cataloged and characterized 204 human and 210 mouse lncRNAs that are differentially expressed following activation of the innate immune response. We have then employed this list of potentially immune modulatory lncRNAs, to identify conserved microdomains and syntenic lncRNAs. To confirm the biological relevance of this analysis, we have shown that the lncRNA IL7-AS [located AS to interleukin-7 (IL7) gene] is induced across multiple human and mouse cell types and regulates the expression and hence the release of the pro-inflammatory mediator, interleukin-6 (IL6).

\section{MATERIALS AND METHODS}

\section{Isolation and Treatment of Human Monocytes and Macrophages}

Human monocytes were prepared as previously described (16). To obtain monocyte-derived macrophages, monocytes were resuspended in MDM complete media [RPMI-1640 supplemented with 10\% (v:v) Fetal Calf Serum, 2 mM L-glutamine, $100 \mathrm{U} / \mathrm{ml}$ penicillin, and $100 \mu \mathrm{g} / \mathrm{ml}$ streptomycin; all GIBCO, Life Technologies] and seeded onto six-well black plates $\left(10^{6}\right.$ cells/ well) for $2 \mathrm{~h}$ at $37^{\circ} \mathrm{C}, 5 \%$ (v:v) $\mathrm{CO}_{2}$ to allow monocytes to adhere to the plate. Non-adherent cells were aspirated, and monocytes were incubated with fresh complete media containing GM-CSF ( $2 \mathrm{ng} / \mathrm{ml} ; \mathrm{R} \& \mathrm{D}$ Systems). Monocytes were incubated at $37^{\circ} \mathrm{C}, 5 \%$ (v:v) $\mathrm{CO}_{2}$ for 12 days to allow full differentiation into MDMs; fresh media containing GM-CSF were replenished on days 4 and 7. Cells were treated with $10 \mathrm{ng} / \mathrm{ml} \mathrm{LPS} \mathrm{for} 4 \mathrm{~h}$, and the controls were left untreated. The media was then removed, and the cells lysed prior to RNA extraction. Circulating blood was collected upon obtaining informed consent, and the study was approved by the National Research Ethics Service (NRES 13/ $\mathrm{LO} / 0354)$.

\section{Production and Treatment of Human Chondrocytes}

For the isolation of primary human chondrocytes, articular cartilage was digested using filter-sterilized collagenase IIA ( $2 \mathrm{mg} / \mathrm{ml}$; Sigma Aldrich) for $5 \mathrm{~h}$ at $37^{\circ} \mathrm{C}$. Digested cartilage was then filtered by passing through a $40-\mu \mathrm{m}$ cell strainer (BD Biosciences), and the filtrate centrifuged. Chondrocytes were then resuspended in growth media [DMEM supplemented with 10\% (v:v) FCS, $2 \mathrm{mM}$ L-glutamine, $100 \mathrm{U} / \mathrm{ml}$ penicillin, $100 \mu \mathrm{g} / \mathrm{ml}$ streptomycin, nonessential amino acids 5\% (v:v); all GIBCO, Life Technologies, 
and $2 \mu \mathrm{g} / \mathrm{ml}$ amphotericin; Sigma Aldrich]. Cells were grown to $70-80 \%$ confluence and, then, either stimulated with $1 \mathrm{ng} / \mathrm{ml}$ IL1 $\beta$ for $4 \mathrm{~h}$, and the controls were left untreated. OA patient joint tissue was collected from the Royal Orthopaedic Hospital (Birmingham) upon obtaining informed consent from patients undergoing elective joint replacement surgery. The study was approved by the NRES (14/ES/1044).

\section{Culture and Treatment of Human Epithelial A549 Cells}

Human epithelial A549 cells were cultured in growth media [DMEM/F-12 supplemented with 10\% (v:v) FCS, $2 \mathrm{mM} \mathrm{L-}$ glutamine, $100 \mathrm{U} / \mathrm{ml}$ penicillin, and $100 \mu \mathrm{g} / \mathrm{ml}$ streptomycin; all GIBCO, Life Technologies] and incubated in a $37^{\circ} \mathrm{C}, 5 \%(\mathrm{v}: \mathrm{v})$ $\mathrm{CO}_{2}$ humidified incubator. For all experiments, A549 cells were seeded in 24-well plate at $1-5 \times 10^{5}$ cell/well and stimulated with $30 \mathrm{ng} / \mathrm{ml}$ IL1 $\beta$ (recombinant, Escherichia coli; Sigma Aldrich) for 4 and $24 \mathrm{~h}$, and the controls were left untreated.

\section{Culture and Treatment of Human Monocytic THP-1 Cells}

THP-1 cells were cultured in growth media [RPMI supplemented with $10 \%$ (v:v) FCS, $2 \mathrm{mM}$ L-glutamine, $100 \mathrm{U} / \mathrm{ml}$ penicillin, $100 \mu \mathrm{g} / \mathrm{ml}$ streptomycin, and $50 \mathrm{nM}$ of 2-mercaptoethanol; all GIBCO, Life Technologies] and incubated in a $37^{\circ} \mathrm{C}, 5 \%$ (v:v) $\mathrm{CO}_{2}$ humidified incubator. For all experiments, THP-1 cells were seeded in 24-well plate at $5-8 \times 10^{5} \mathrm{cell} /$ well and stimulated with $1 \mu \mathrm{g} / \mathrm{ml}$ LPS (E. coli 055:B5; Sigma Aldrich) for 4 and $24 \mathrm{~h}$, and the controls were left untreated.

\section{Culture and Treatment of Mouse RAW 264.7 Macrophages}

RAW 264.7 cells were cultured in growth media [DMEM supplemented with $10 \%$ (v:v) FCS, 2 mM L-glutamine, $100 \mathrm{U} / \mathrm{ml}$ penicillin, and $100 \mu \mathrm{g} / \mathrm{ml}$ streptomycin; all GIBCO, Life Technologies] and incubated in a $37^{\circ} \mathrm{C}, 5 \%$ (v:v) $\mathrm{CO}_{2}$ humidified incubator. For all experiments, RAW cells were seeded in 24-well plate at $2-5 \times 10^{5}$ cell/well and stimulated with $1 \mu \mathrm{g} / \mathrm{ml} \mathrm{LPS}$ (E. coli 055:B5; Sigma Aldrich) for 4 and $24 \mathrm{~h}$, and the controls were left untreated.

\section{RNA Isolation and Quality Control}

For all samples, total RNA was extracted using the RNeasy kit (Qiagen), included an on-column DNase treatment (Qiagen), according to the manufacturer's guideline. RNA concentration was determined using the Qubit 2.0 (Life Technologies). RNA quality was measured using the Agilent Bioanalyser and produced RIN values $>8.0$.

\section{RNA Library Preparation and Sequencing}

Total RNA from epithelial A549 and RAW 264.7 cells were purified using polyA + fractionation (Illumina), while the monocytes, macrophages, and synovial chondrocytes were subjected to ribosome depletion (Ribo-Zero, Illumina). For all tissues, cDNA libraries were prepared using the Illumina TruSeq Stranded Total RNA kit. Samples were then subjected to $100 \mathrm{bp}$, paired-end sequencing upon an Illumina 2000 or 2500 sequencing machine (Wellcome Trust Sequencing Unit, University of Oxford). Quality scores across sequenced reads were assessed using FASTQC v0.9.2. ${ }^{1}$ All samples were of high quality with the average score (mean and median) at each base across reads in each sample $Q>35$. Historical mouse sequencing data were download from Sequence Read Archive (SRA) ${ }^{2}$ using the following command in SRA tools: fastq-dump -I --split-files < file_name>. This included data on bone marrow-derived macrophages (BMDMs) (ribozero, paired-end, and non-stranded, $n=2$ ) (19), peritoneal macrophages, and splenic dendritic cells (ribozero, paired-end, and non-stranded, $n=2)(21)$.

\section{Alignment and Assembly of Human and Mouse IncRNAs}

Paired-end reads were aligned to the human reference genome (hg38) using TopHat2 (version 2.1.0) (22) or the mouse reference genome (mm 10) using Hisat2 (version 2.0.4) (23) using the following command line options. Tophat2: tophat --library-type fr-firststrand $<$ reference_genome.gtf $>-1<$ forward_strand.fa $>$ $-2<$ reverse_strand.fa $>-o<$ output.sam $>$. Hisat2: hisat $2-\mathrm{q}$ --dta --rna-strandness FR $-\mathrm{x}<$ reference_genone.gtf $>-1$ $<$ forward_strand.fa $>-2<$ reverse-strand file.fa $>-S<$ output. sam $>$. Output SAM files were then sorted and converted to BAM files (samtools sort -@8-o output.bam output.sam) and indexed (samtools index -b output.bam) in Samtools (24). The BAM output files for all control and LPS or IL1 $\beta$ samples were merged using Bamtools (25) to produce two files per cell type. All possible genes from these two BAM files were assembled $a b$ initio using StringTie $(26,27)$ using the following command line options: stringtie <input.bam>-o assembled_genes.gtf -e -A gene_quantification.txt. The eight GTFs containing the genes from across the four cell types (both control and activated) were then combined using Cuffmerge v2.2.1.0 (which is part of the Cufflinks suite) (28) to produce a "total" GTF containing all possible genes and converted into a BED file. The single and multiple exon genes were separated using the information obtained in column 10 (block/exon), and those genes $<200$ nucleotides were removed using the information in column 11 (exon lengths). The resulting two BED files containing single exon and multi-exonic genes were compared with Gencode v23 (29) using BEDtools 2 (30) to identify known and novel lncRNAs. Potential protein-coding genes were identified using the coding potential calculator ${ }^{3}(31)$. The GTF containing novel single and multi-exonic lncRNAs was concatenated with the Gencode v23 catalog (29), to produce a "master" human GTF employed for gene quantification using CuffNorm, Stringtie, and CuffDiff. Parallel analysis of the expression of proteincoding genes and lncRNAs in mouse was undertaken using Gencode m12 (32).

${ }^{1}$ http://www.bioinformatics.babraham.ac.uk/projects/fastqc.

${ }^{2}$ https://www.ncbi.nlm.nih.gov/sra.

${ }^{3}$ http://cpc.cbi.pku.edu.cn. 


\section{Principle Component Analysis and Hierarchical Clustering}

The abundance of potential lncRNAs and Gencode v23 defined genes in individual samples was defined as the fragments per kilobase exon per million reads mapped (FPKM) and determined using CuffNorm v2.2.1.1 (part of the Cufflinks suite) (28). PCA and hierarchical clustering on Gencode v23 genes demonstrating an expression $>1$ FPKM were performed using Genesis (v1.7.7) (33). Data were $\log 2$ transformed following the addition of 1 FPKM. The threshold for reporting gene expression at FPKM $>1$ is based upon the ability to validate sequencing data using qRTPCR (34).

\section{Differential mRNA and IncRNA Expression}

The differential expression of assembled lncRNAs and Gencodeannotated protein-coding genes was assessed with the geometric option (DESeq) in Cuffdiff v2.2.1.3 (part of the Cufflinks suite) (28) using a significance threshold of $q<0.05$. The command line options were as follows: cuffdiff --FDR $=0.05$--min-alignmentcount $=10$--library-norm-method $=$ geometric - -dispersionmethod $=$ pooled $-\mathrm{u}<$ reference_genome.gtf $><$ control_1. bam $>,<$ control_x.bam $>$, < activated_1.bam $><$ activated_x. bam $>-0<$ output_file_name $>$.

\section{Assembly of IncRNA Gene Sequences}

A BED file containing all the transcripts for each lncRNAs was extracted from the "master" GTF files and the exons extracted using the Gene BED to Exon/Intron/Codon BED expander (at www.usegalaxy.org) (35). Overlapping exons (genomic coordinates) from each transcript were merged using Bedtools 2 (30), the relevant DNA sequences were extracted using Extract Genomic DNA (at www.usegalaxy.org) (35), and all exons sequences merged to produce a FASTA of the lncRNA gene sequences.

\section{Determination of Evolutionary Conservation}

A BED file containing all the transcripts for each lncRNAs was extracted from the master GTF files and submitted into the Table Browser Tool on the UCSC genome browser for comparative genomics $^{4}(36)$.

\section{Identification and Removal of Repeat Sequences}

Repeat sequences were identified and removed from the assembled lncRNA sequences (FASTA) using the default options in Repeatmasker. $^{5}$

\section{Identification of Conserved Microdomains}

Potential motifs within the lncRNA genes were identified by submitting gene sequences (FASTA) into MEME-ChIP option on MEME-Suite (37). Identification of conserved sequences between lncRNAs was undertaken with BLAST + (38) by input of the FASTA files as both query and subject using the following command line: blastn -query <query_file.fasta> -subject $<$ subject_file.fasta> -task blastn -outfmt "6 qacc sacc sseq pident qlen length evalue" $>$ output.txt. These were compared with random control sequences of comparable lengths and AT ratios generated in the Build Controls Section (random sequences) of RSAT $^{6}$ (39), while random protein-coding sequences of comparable lengths were selected from the mRNA sequences downloaded via Biomart in Ensembl. ${ }^{7}$ Output from all these BLASTn analyses (FASTA) was then submitted to MEME-ChIP.

\section{Identification of Syntenic IncRNAs}

To identify syntenic lncRNA in the human and mouse catalogs, we used the Liftover program ${ }^{8}$ (36) to identify the predicted position of the human lncRNAs (hg38) on the mouse genome (mm10) and then examined whether these overlapped with the assembled mouse lncRNAs using Bedtools 2 (30).

\section{ChIPseq Analysis}

ChIPseq sequencing files (FASTQ) containing information on $\mathrm{H} 3 \mathrm{~K} 27 \mathrm{ac}$ and H3K4me3 deposition and related input controls ( $n=2$ per condition), from control and LPS-stimulated human monocytes at $4 \mathrm{~h}$ (GSE85245) (40), were download from SRA (see text footnote 2) using SRA tools: fastq-dump < file_name>. Sequencing data were aligned to hg38 using Bowtie 2 (41): bowtie 2 -q --very-fast <reference_genome.gtf $>-U<$ file_name. fastq $>-S<$ output_file.sam $>$. Output SAM files were then sorted and converted to BAM files (samtools sort -@8-o output. bam output.sam), indexed (samtools index -b output.bam) in Samtools (24), and then converted to BigWig format using BamCoverage, which is part of the deepTools suite (42) using the following command line: bamCoverage -b <input_bam. bam> --normalizeUsingRPKM --binSize 30 --smoothLength 300 -p 10 --extendReads 200 -o <output_file.bw>. Significant ChIPseq peaks $(n=2$ pre-condition and $q=<0.1)$ were called with MACS2 (43) using the broadpeak options: macs2 callpeak - $t$ $<$ sample_1 > < sample_2 > -c < control_1 > < control_2 > -broad $<$ output_files $>-\mathrm{g}$ hs. The intersection between ChIPseq data (broadpeak.bed) and mRNA and lncRNA was undertaken using the Join option in the Operate upon Genomic Intervals section of Galaxy (at www.usegalaxy.org) (35). Heatmaps of the data were generated using deepTools. Matrices containing summary scores around promoters were generated from the $\mathrm{H} 3 \mathrm{Kme} 3$ and $\mathrm{H} 3 \mathrm{~K} 27 \mathrm{ac}$ BigWig files using the following options in the computeMatrix tool: computeMatrix reference-point $-S<$ filename. bw $>$-R <mRNA/lncRNA.bed $>$-b 3000 -a 3000 -out <matrix. name $>$. Heatmaps were then generated using the plotHeatmap function and the following options: plotHeatmap $-\mathrm{m}<$ matrix. name $>$--colorMap YlOrRd --samplesLabel " $<$ Sample Names $>$ " -out $<$ plot_name.eps $>$.

${ }^{6} \mathrm{http}: / /$ rsat.sb-roscoff.fr

${ }^{7}$ http://www.ensembl.org/index.html.

${ }^{8} \mathrm{http}: / /$ genome.ucsc.edu/cgi-bin/hgLiftOver. 


\section{Nuclear-Cytoplasm RNA Fractionation}

A549 and THP-1 cells were stimulated with IL1 $\beta$ (30 ng/ml) or LPS $(1 \mu \mathrm{g} / \mathrm{ml})$ for $4 \mathrm{~h}$ respectively. The cells were scraped (i.e., A549) and/or collected, then centrifuged $(12,000 \times g$, $\left.1 \mathrm{~min}, 4^{\circ} \mathrm{C}\right)$. Supernatants were discarded, and the pellets resuspended in $1 \mathrm{ml}$ of cold $\mathrm{PBS}$ and then split into two equal fractions (500 $\mu \mathrm{l}$ each). Fractions were then centrifuged, and their supernatants discarded. One of the two fractions was resuspended and lysed in $350 \mu$ l of RLT buffer (Qiagen) and constituted the whole lysate fraction. The pellet of the remaining fraction was resuspended in $175 \mu \mathrm{l}$ of cold RLN buffer [50 mM Tris pH 8, $140 \mathrm{mM} \mathrm{NaCl}, 1.5 \mathrm{mM} \mathrm{MgCl}_{2}, 0.5 \%$ (v:v) Non-idet P-40, $0.5 \mathrm{mM}$ DDT, $1 \times$ Halt protease inhibitor cocktail; Thermo Fisher, and 20 U/ml SUPERase-IN; Ambion] and incubated on ice for $\sim 15 \mathrm{~min}$, in order to lyse the plasma membrane while leaving the nuclei intact. The nuclei were then isolated by centrifugation $\left(300 \times g, 10 \mathrm{~min}, 4^{\circ} \mathrm{C}\right)$. The supernatant was delicately collected and transferred into a fresh tube, and $600 \mu \mathrm{l}$ of RLT buffer was added to it to constitute the cytoplasmic fraction while, the pellet was resuspended in $350 \mu \mathrm{l}$ of RLT buffer to form the nuclear fraction. Total RNA was then extracted, and cDNA libraries were made using a set volume of RNA determined by the whole lysate fraction RNA quantity, within individual experiments. mRNA and RNA expressions were then determined using qPCR, and results were expressed as fold change compared to whole lysate stimulated samples.

\section{Transfection of THP-1, RAW 264.7, and A549 Cells with AS Locked Nucleic Acid GapmeRs}

The following protocol was used to transfect all cell types (i.e., human monocytic THP-1 cells, mouse macrophage RAW 264.7 cells, and human epithelial A549 cells). AS Locked Nucleic Acid (LNA) GapmeRs (Exiqon, sequences are listed in Table S1 in Supplementary Material, final concentration of $30 \mathrm{nM}$ ) were mixed in $100 \mu \mathrm{l}$ of serum- and antibiotic-free medium, supplemented with $5 \mu \mathrm{l}$ of HiPerFect (Qiagen). Each mix was then added to each well of a 24-well plate. Cells were resuspended at desired concentration $\left(1-8 \times 10^{5}\right.$ cells/well $)$ in $100 \mu \mathrm{l}$ of their corresponding complete medium and added on top of the LNA GapmeRs mixes. Cells were then incubated for $16 \mathrm{~h}$. The following day cells were diluted with $400 \mu \mathrm{l}$ of complete medium and stimulated with either LPS or IL1 $\beta$. Cells' supernatants were then collected for the analysis of cytokine release (see ELISA), and cells were lysed for RNA extraction at 4 and $24 \mathrm{~h}$. mRNA and RNA expressions were then determined using $\mathrm{qPCR}$, and results were expressed as fold change compared to non-transfected stimulated samples.

\section{Cytokine Release Measurement by ELISA}

Following stimulation of A549, THP-1, and RAW 264.7 cells, supernatants were collected and measurements of human and mouse IL6 were made by ELISA (R\&D Systems) according to the manufacturer's guidelines. Results are expressed in percentage of maximum response of control stimulation.

\section{Availability of Data and Materials}

All software, including the web address of the source code, is listed in Table S2 in Supplementary Material. The sequencing data are available from the gene expression omnibus under the following entries: human monocytes (ERA294222), human macrophages (GSE101868), human chondrocytes (GSE74220), human lung epithelial A549 cells (GSE101868), mouse RAW 264.7 macrophages (GSE101868), BMDMs (PRJEB11889), peritoneal macrophages, and splenic dendritic cells (SRP038980).

\section{RESULTS}

\section{Differential Expression of Protein-Coding Genes following Activation of the Human Innate Immune Response}

We undertook stranded and paired-end sequencing data on total RNA obtained from four activated human cell types associated with the innate immune response including two myeloid immune cells, monocytes (ERA294222) and monocyte-derived macrophages (macrophages) (GSE101868), and two stromal cell types, lung A549 epithelial cells (GSE101868) and synovial chondrocytes (GSE74220). The myeloid cells were activated with bacterial LPS (via TLR4), while the stromal cells were activated using the pro-inflammatory cytokine, IL1 $\beta$. Sequencing produced 2.0 billion reads ( 100 bases per read) of which $87 \%$ could be aligned to the human reference genome. Principle component analysis and unsupervised hierarchical clustering of the mRNA expression data (>1 FPKM) demonstrated separation of control and activated monocytes, macrophages, chondrocytes, and epithelial cells (Data Sheet S1 in Supplementary Material). Using the Gencode database (v23), we showed differential expression $(q<0.05)$ of 1,955 mRNAs in monocytes, 1,386 mRNAs in macrophages, 1,708 mRNAs in epithelial cells, and 855 mRNAs in chondrocytes (Table S3 in Supplementary Material). Integration of the data identified 3,853 mRNAs that were differentially expressed across all cell types. Of these, 2,479 (65\%) were expressed in a single cell type, 858 (22\%) were expressed in two cell types, 347 (9\%) were expressed in three cell types, and 166 (4\%) were expressed in four cell types (Figure 1A). As might be expected, KEGG pathway analysis (using DAVID bioinformatics platform) (44) showed that the 2,359 differentially up-regulated mRNAs across all four cell types were associated with activation of the innate immune response (Figure 1B). In contrast, the 1,494 down-regulated mRNAs were not associated with any pathways. These data indicated activation of the innate immune response in all four cell types following exposure to either of the proinflammatory mediators, LPS or IL1 $\beta$.

\section{Profile of IncRNA Expression in Resting Human Cells}

We identified 1,162 lncRNA genes that contained at least two exons and were expressed at $>1$ FPKM in at least one cell type (either control or stimulated cells) (Figure 1C; Table S4 in Supplementary Material). For clarity, we have included the " $h$ " and " $m$ " prefixes to identify human and mouse lncRNAs. Of 


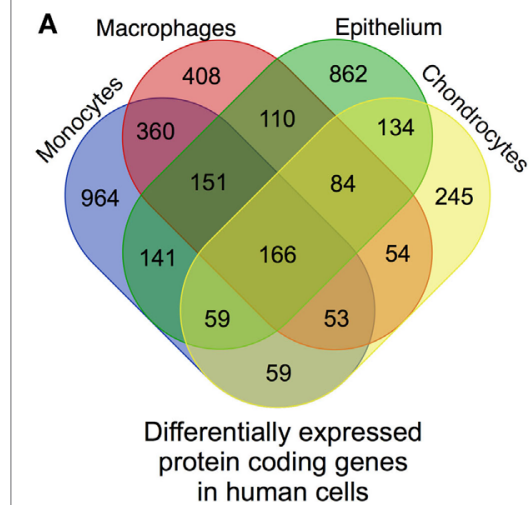

C

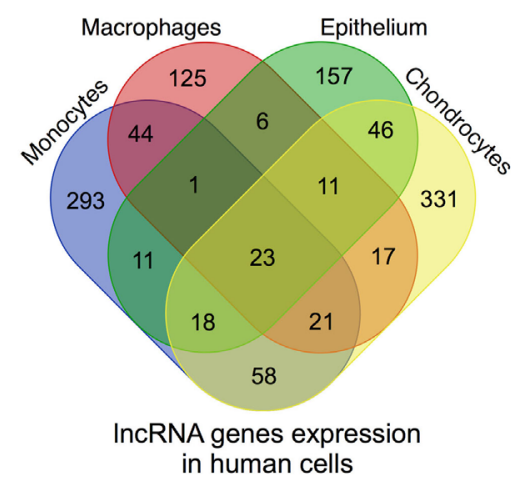

B

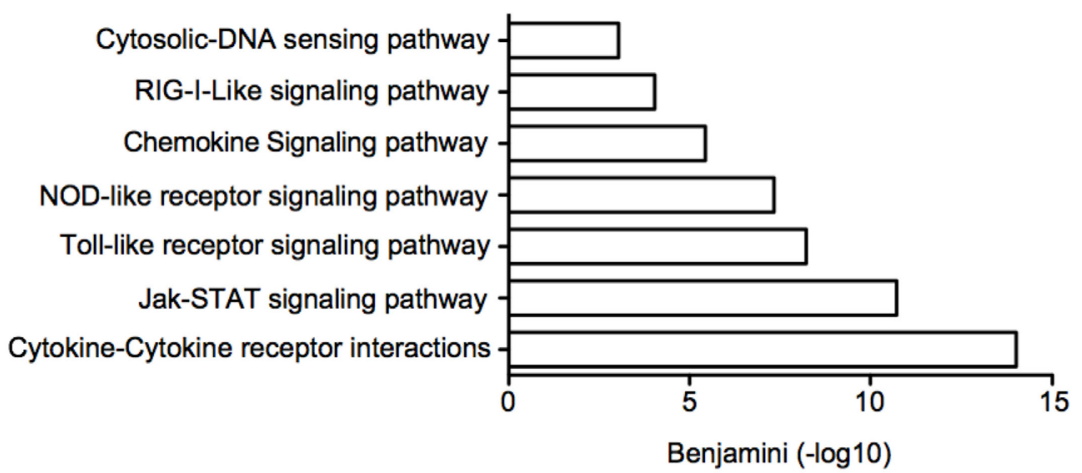

D

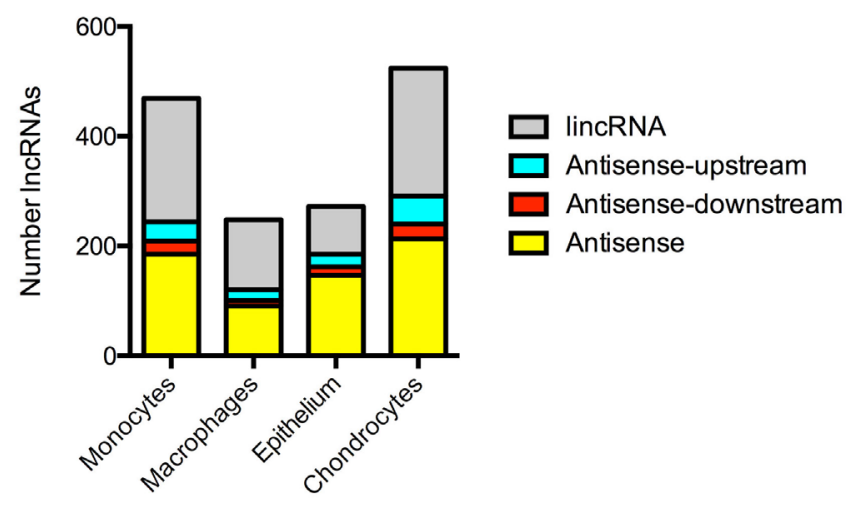

E SINES (Alu/MIR)

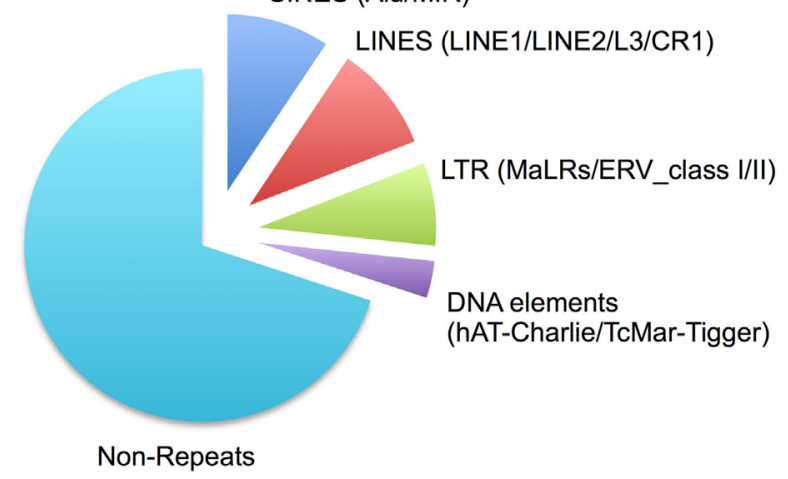

FIGURE 1 | Profile of messenger RNAs' (mRNAs) and long non-coding RNAs' (IncRNAs) expressions in human cells. (A) Venn diagram showing the overlap in the differentially expressed mRNAs following lipopolysaccharides (LPS)-induced activation of monocytes and macrophages or IL $1 \beta$-induced activation of epithelial cells and chondrocytes at $4 \mathrm{~h}$. (B) Pathways analysis of the mRNAs that were differentially expressed across all human cell types. (C) Venn diagram showing the overlap in the IncRNAs expression profile in resting monocytes, macrophages, epithelial cells, and chondrocytes. (D) Distribution of different antisense and lincRNA species in resting monocytes, macrophages, epithelial cells, and chondrocytes and (E) Pie chart showing the percentage distribution of repeat sequences in the total IncRNA population obtained from all cell types.

these assembled genes, 586 overlapped with lncRNAs annotated in Gencode v23, meaning that the remaining 576 (50\%) likely represent novel lncRNAs. Detailed breakdown identified 469 lncRNAs (54\% novel) in monocytes, 248 lncRNAs (40\% novel) in macrophages, 273 lncRNAs (32\% novel) in epithelium, and 526 lncRNAs (44\% novel) in chondrocytes. As in previous reports, we divided lncRNAs into four groups based upon their relative position to protein-coding genes: AS (overlapping a protein-coding 
gene on the opposite strand), AS-upstream (within $5 \mathrm{~kb}$ and located upstream/opposite strand from of a protein-coding genes), AS-downstream (within $5 \mathrm{~kb}$ and located downstream/ opposite strand from of a protein-coding genes), and lincRNAs (located $>5 \mathrm{~kb}$ from a protein-coding gene) (16). We have excluded lncRNAs located on the same strand and within $5 \mathrm{~kb}$ of a protein-coding gene, since these could potentially represent gene extensions. Using these criteria, it was found that lncRNAs could be subdivided into 39\% AS, 5\% AS-downstream, 8\% AS-upstream, and $48 \%$ lincRNA, and this ratio remained similar across the four cell types (Figure 1D).

Examination of the overlap showed that the vast majority of lncRNA were expressed in a cell-specific manner with 906 (78\%) selectively expressed in a single cell type, $182(16 \%)$ in two cell types, 51 (4\%) in three cell types, and 23 (2\%) in all four cell types (Figure 1C; Table S4 in Supplementary Material). As previously reported (45), the lncRNAs were found to be enriched with repeat sequences (identified using repeatmasker.org) including 9.4\% short interspersed nuclear elements (SINES), 9.7\% long interspersed nuclear elements (LINES), 7.6\% long terminal repeats (LTRs), and 3.4\% DNA elements, leaving $70 \%$ of non-repeat sequence (Figure 1E).

We have identified 1,162 lncRNAs across the four human cell types including 576 novel lncRNAs that were enriched in repeat sequences and expressed in a predominantly cell-specific manner.

\section{Widespread Differential Expression of IncRNAs following Activation of the Human Innate Immune Response}

To identify lncRNAs that might regulate the innate immune response, we examined their differential expression following exposure to either LPS (monocytes and macrophages) or IL1 $\beta$ (epithelium and chondrocytes) (Table S5 in Supplementary Material). We showed differential expression of 105 lncRNAs in monocytes, 50 lncRNAs in macrophages, 39 lncRNAs in epithelium, and 65 lncRNAs in chondrocytes (Figures 2A,B; Table S5 in Supplementary Material). This produced a total of 204 differentially expressed lncRNAs, which could be subdivided into 127 lincRNAs (62\%), 45 AS (22\%), 17 AS-downstream (8\%), and 15 AS-upstream (8\%). Comparison with Gencode v23 showed that 93 lncRNAs overlapped with annotated genes, indicating that the remaining 111 might be novel (Table S5 in Supplementary Material). Evaluation of the absolute change in expression across the four cell types showed a 10-fold difference between differentially expressed mRNAs and lncRNAs, with a mean $( \pm$ SEM $)$ of $55.5 \pm 3.1$ FPKM and $5.4 \pm 3.1$ FPKM, respectively. As with mRNAs, examination of the overlap between cell types showed that the vast majority (161 or 79\%) were differentially expressed in a cell-specific manner (Figure 2B). Of the remainder, 33 (16\%) were found in two cell types, eight (4\%) in three cell types, and only two (1\%) in all four cell types (Figure 2B).

Once again, large regions of repetitive sequences were found in AS (33\%), AS-downstream (39\%), AS-upstream (25\%), and lincRNA (33\%) (Figure 2C). To assess their potential function, we identified 699 genes located within $1 \mathrm{Mb}$ of these differentially expressed lncRNAs using GREAT ${ }^{9}$ and showed that these were associated with immune activation and response (Figure 2D). Comparison of the fold change showed a correlation between expression of the nearest mRNA expression and that of the AS $(r=0.495, p<0.0001)$, AS-downstream $(r=0.567, p<0.0001)$, AS-upstream $(r=0.760, p<0.0001)$, and lincRNAs $(r=0.520$, $p<0.0001$ ) (Figure 2E), which was not seen when we looked at the total lncRNA population (Data Sheet S2 in Supplementary Material).

In summary, we identified 204 lncRNAs that were differentially expressed across the four human cell types (including 111 novel lncRNAs) that could be subdivided into 62\% lincRNAs, $22 \%$ AS, and $8 \%$ AS-downstream and $8 \%$ AS-upstream. The majority (161 lncRNAs) were expressed in a cell-specific manner, although there were $43 \mathrm{lncRNAs}$ that were induced in multiple cell types. Positional analysis showed that lncRNA expression was correlated with immune-related genes and suggested that these might be functionally linked.

\section{Differential Expression of Single Exon IncRNAs during Activation of the Human Innate Immune Response}

Although we had included only multi-exonic genes in our initial analysis, a number of the previous publications have identified single exon lncRNAs that regulate the innate response, including PACER (13) and THRIL (14). To provide a complete picture of the role of non-coding RNAs in the innate immune response, we therefore decided to include these in the analysis. Our $a b$ initio assembly identified 44,656 single exon lncRNAs genes that were $>200 \mathrm{nt}$ and expressed at $>1$ FPKM in at least one cell type (either control or stimulated cells) (Table S6 in Supplementary Material). Significantly, the vast majority (42,085 or $94 \%$ ) showed no overlap with annotated lncRNAs in Gencode v23, while breakdown by cell type identified 8,068 lncRNAs in monocytes, 313 lncRNAs in macrophages, 2,657 lncRNAs in epithelium, and 37,829 lncRNAs in chondrocytes. The wide variation between cell types indicated that identification might be influenced by sequencing variability and that many of these single exons lncRNAs likely represent artifacts.

With these reservations in mind, we proceeded to identify those that were differentially expressed following activation of the innate immune response. Once again there was wide variation between cells with 510, 216, 33, and 710 differentially expressed IncRNAs in monocytes, macrophages, epithelium, and chondrocytes, respectively (Figure $\mathbf{3 A}$ ). This produced a combined total of 1,250 lncRNAs across all four cell types, of which only a small proportion $(3.5 \%)$ were shown to overlap with annotated IncRNAs in Gencode v23. Significantly, unlike the multi-exonic lncRNAs, only 24 of the 1,250 differentially expressed single exon lncRNAs were identified in two cell types and none were expressed in three or four cell types.

Using the percentage distribution across individual cell types, these were shown to be predominantly lincRNAs (78\%), with

${ }^{9}$ http://bejerano.stanford.edu/great/public. 


\section{A}

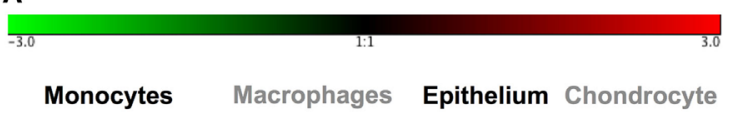

Control LPS Control LPS Control IL1 $\beta$ Control IL1 $\beta$

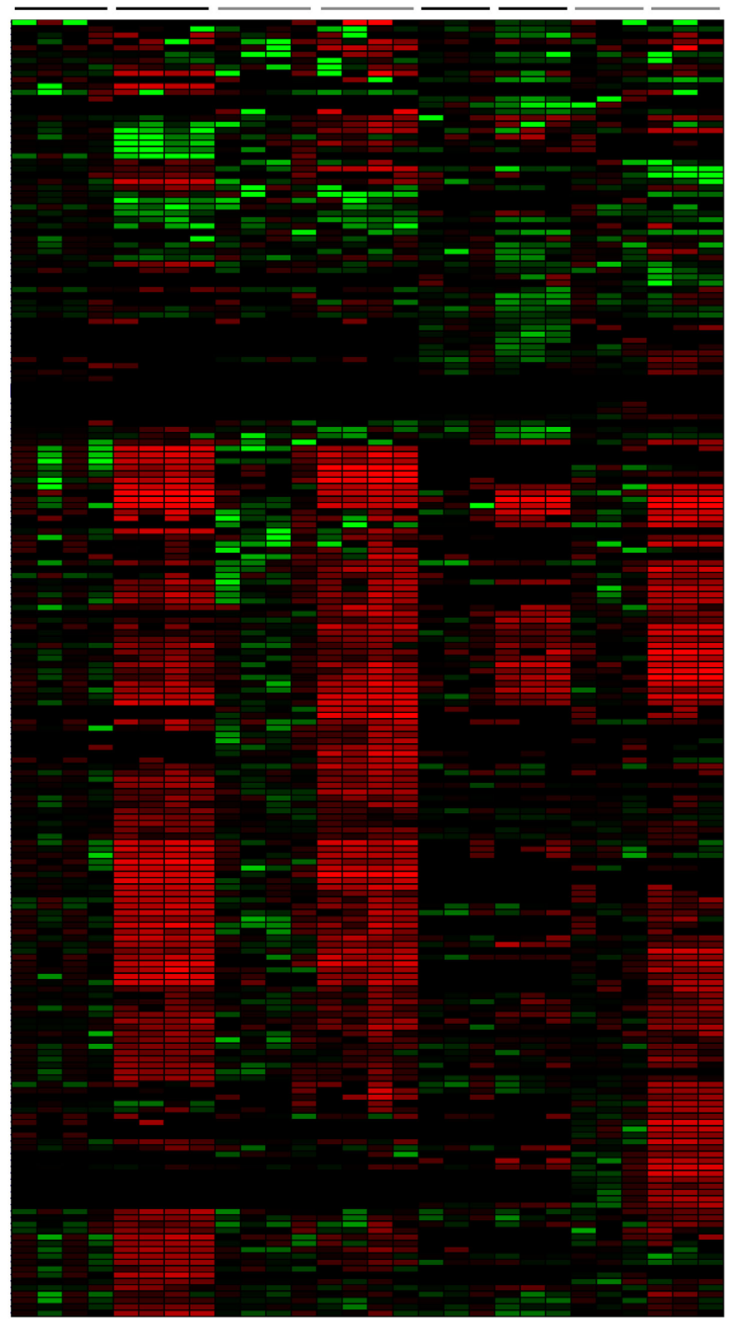

D

GO Biological Process

$-\log 10$ (Binomial $p$ value)

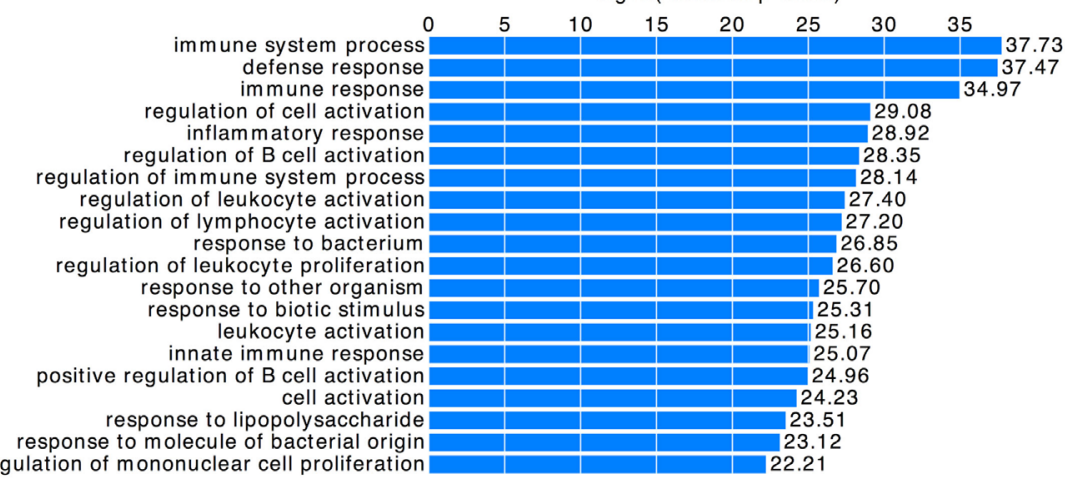

E

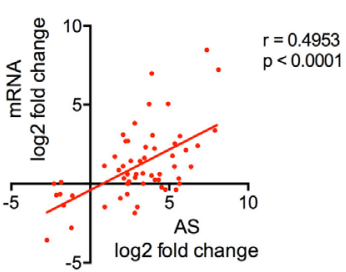

\section{Antisense-Upstream}

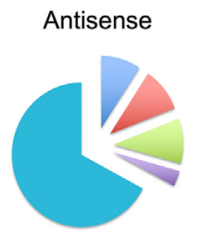

Antisense-Downstream

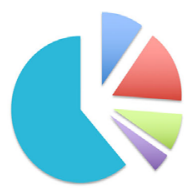

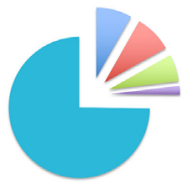

lincRNA
- SINE

- LINES

- LTR elements

- DNA elements

- Non-repeats
Antisense-Downstream

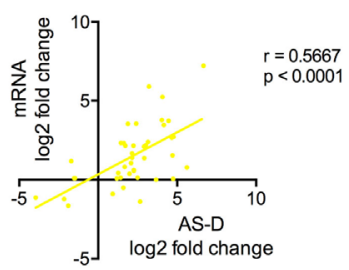

Antisense-Upstream

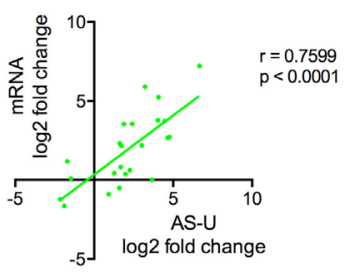

lincRNA

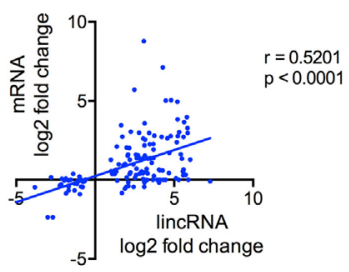

FIGURE 2 | Continued 


\section{FIGURE 2 | Continued}

Characterization of differentially expressed long non-coding RNAs (IncRNAs) following activation of the human innate immune response. (A) Heatmap of the IncRNA expression levels in control and lipopolysaccharides (LPS) or interleukin-1 $\beta$ (IL1 $\beta$ ) stimulated monocytes, macrophages, epithelium, and chondrocytes that have been subjected to unsupervised hierarchical based by experiment. (B) Venn diagram showing the overlap in the differentially expressed IncRNAs in LPS-stimulated monocytes and macrophages and IL1 $\beta$-stimulated epithelial cells and chondrocytes at $4 \mathrm{~h}$. (C) Pie charts showing the percentage distribution of repeat sequences in the various sub-populations of the differential expression IncRNAs across all four cell types with SINES = short interspersed nuclear elements, LINES = long interspersed nuclear elements, and LTR = long terminal repeat. (D) Pathways analysis of the messenger RNAs (mRNAs) located within 1 Mb of the differentially expressed IncRNA. (E) Pearson's correlation between the differential expression of various IncRNA populations and that of the nearest mRNA.

much smaller numbers of AS (3\%), AS-downstream (7\%), and AS-upstream (12\%) (Figure 3B). As might be expected, these single exon $\operatorname{lncRNAs}$ were shorter in length than the multi-exonic lncRNAs at $0.6 \mathrm{~kb}(1.9 \mathrm{~kb} ; p<0.0001), 1.0 \mathrm{~kb}(1.5 \mathrm{~kb} ; p=0.0039)$, and $2.1 \mathrm{~kb}(2.4 \mathrm{~kb} ; p<0.0001)$ for AS-downstream, AS-upstream, and lincRNA, respectively (numbers in brackets show the length for multi-exonic lncRNAs; paired statistical testing using Mann Whitney). The exception was the AS at $3.6 \mathrm{~kb}$, which were longer than the $2.9 \mathrm{~kb}$ seen in multi-exonic AS ( $p<0.0001)$. Comparison of the absolute change in expression gave a value of $3.0 \pm 0.3$ FPKM, a value not significantly different from 5.4 \pm 3.1 FPKM seen with multi-exonic lncRNAs (Kruskal-Wallis test).

Once again, these single exon lncRNAs were also composed of large regions of repetitive sequences, which comprised 36, 31, 26 , and $29 \%$ of the AS, AS-downstream, AS-upstream, and lincRNA sequences, respectively (Figure 3C). Assessment of their potential function identified 2,256 genes located within $1 \mathrm{Mb}$ of these differentially expressed single exon lncRNAs and showed that these were also associated with immune activation and response (Figure 3D) and with the exception of AS-downstream that changes in expression correlated with those of the nearest mRNA (Figure 3E).

We were able to identify large numbers of differentially expressed single exon lncRNAs, whose expression was strongly cell type specific and correlated with that of adjacent immunerelated genes. However, we speculate that the vast majority represent artifacts related to the computational analysis and/or local non-specific transcriptional activity. Interestingly, although we showed significant increases in the expression of PACER ( $h X L O C \_015084$ ) in monocytes and chondrocytes (Table S6 in Supplementary Material), we were unable to detect the presence of THRIL (14). This is purported to be embedded (in the AS direction) within the $3^{\prime}$-untranslated region (UTR) of $B R I 3 B P$ but detailed visual inspection in monocytes and macrophages (as well as the other two cell types) failed to identify the presence of this IncRNA (Data Sheet S3 in Supplementary Material).

\section{LncRNA Expression in Human Monocytes Correlates with Activating Histone Marks}

To validate our lncRNA catalog, we examined in control and LPS-stimulated monocytes the overlap between the lncRNAs and two active histone marks; $\mathrm{H} 3 \mathrm{~K} 4 \mathrm{me} 3$, a marker of transcriptional activity, and $\mathrm{H} 3 \mathrm{~K} 27 \mathrm{ac}$, a marker of active promoters and enhancers (40). Intersection of the peaks identified by MACS2 and the mRNAs expressed in resting monocytes showed a partial overlap with H3K4me3 (25\%) (Figure 4A) and H3K27ac (20\%) (Figure 4B). In comparison, the overlap between the multi-exonic
lncRNAs and $\mathrm{H} 3 \mathrm{~K} 4 \mathrm{me} 3$ was reduced at $10 \%$, while there was an increased intersection with the deposition of $\mathrm{H} 3 \mathrm{~K} 27 \mathrm{ac}$ at $27 \%$. By contrast, there was little overlap between the single exon lncRNAs and $\mathrm{H} 3 \mathrm{~K} 4 \mathrm{me} 3$ (1\%) or H3K27ac (4\%).

To examine the differentially expressed lncRNAs, we subsequently focused on the peaks identified following the same length of LPS stimulation in monocytes (4 h). As might be expected, the overlap between mRNAs and H3K4me3 (59\%) (Figure 4C) and H3K27ac (62\%) (Figure 4D) was greatly increased compared with resting cells and was comparable to the intersection seen with lncRNAs (H3K4me3 70\% and $\mathrm{H} 3 \mathrm{~K} 27 \mathrm{ac} 75 \%)$. In the case of differentially expressed single exons lncRNAs, the overlap was increased compared to controls (H3K4me3 24\% and H3K27ac 44\%) but did not reach the levels in mRNAs and lncRNAs. We further examined the profile of the two marks across the promoters $( \pm 3 \mathrm{~kb})$ of the differentially expressed mRNAs and lncRNAs (Figures 4E,F). Although we were unable to detect a global increase in $\mathrm{H} 3 \mathrm{~K} 4 \mathrm{me} 3$ and $\mathrm{H} 3 \mathrm{~K} 27 \mathrm{ac}$ at the promoters of mRNAs (Figure 4E), the deposition of both H3K4me3 and H3K27ac was clearly increased for both the lncRNAs and single-exonic lncRNAs (Figure 4F). Overall, this ChIPseq analysis provides additional evidence to support our transcriptional analysis showing LPS-induced expression of multi-exonic lncRNAs and, to a lesser extent, single exon lncRNAs, in human monocytes. This also supports the existence of lncRNAs in resting monocytes, although the poor overlap with single exon lncRNAs indicates that many are indeed artifacts.

\section{Widespread Differential Expression of IncRNAs following Activation of the Mouse Innate Immune Response}

Further studies were undertaken to identify and characterize the differentially expressed lncRNAs following activation of the mouse innate immune response and to compare these with human lncRNAs. To this end, we undertook sequencing of LPSstimulated mouse RAW 264.7 macrophages and combined this with published sequencing data obtained from LPS-stimulated BMDMs (19), LPS-stimulated peritoneal macrophages (21), and LPS-stimulated splenic dendritic cells (21). Using the mouse Gencode database (m12), we showed differential expression $(q<0.05)$ of 1,293 mRNAs in BMDMs, 1,487 in RAW 264.7 macrophages, 90 in peritoneal macrophages, and 24 in dendritic cells (Figure 5A; Table S7 in Supplementary Material). As with the human cells, KEGG pathway analysis (using DAVID bioinformatics platform) (44) indicated activation of the immune response in all four cell types following exposure to LPS (Figure 5B). 
A

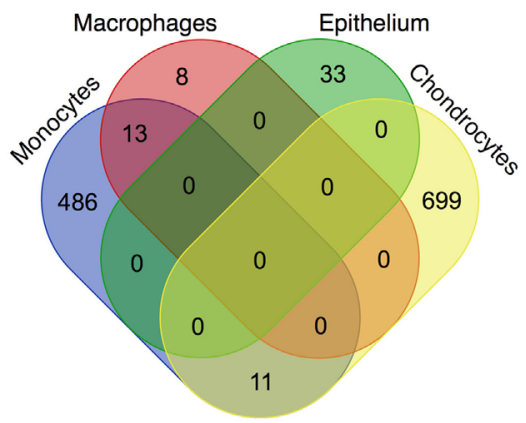

Differentially expressed single exons IncRNA in human cells

B

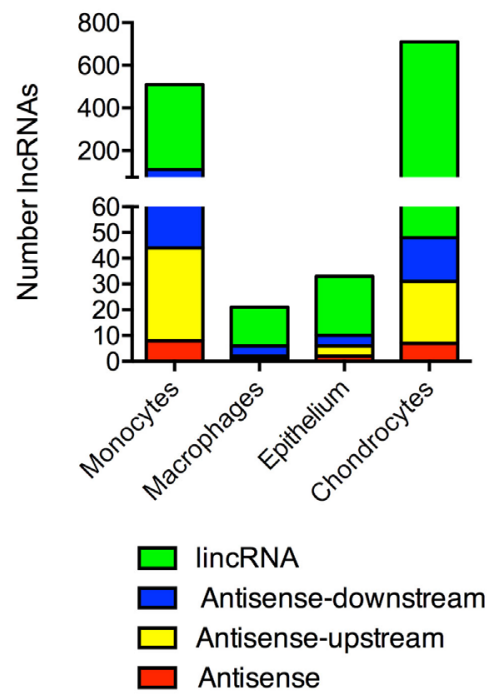

D

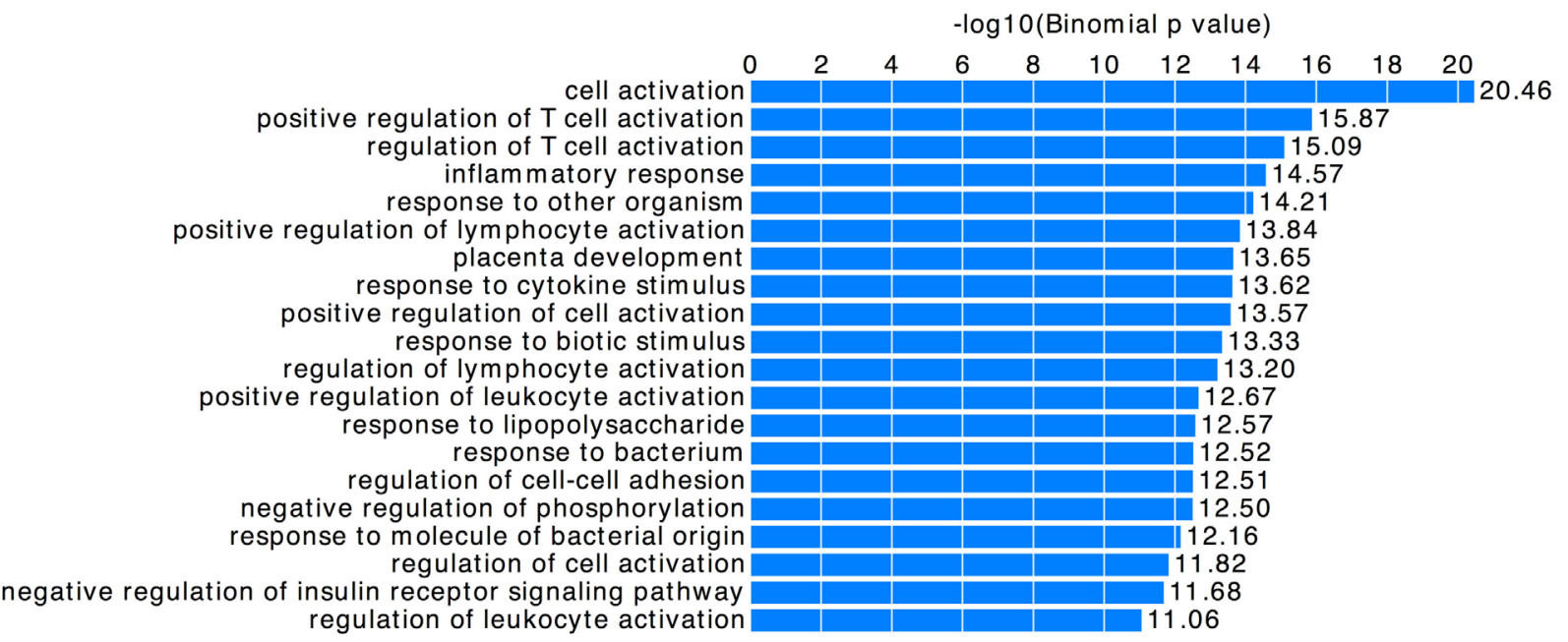

C

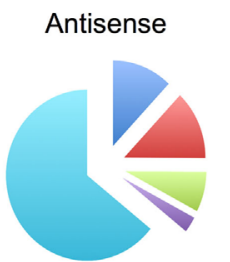

Antisense-Downstream

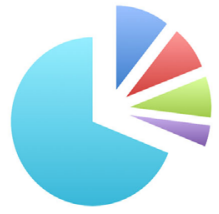

E

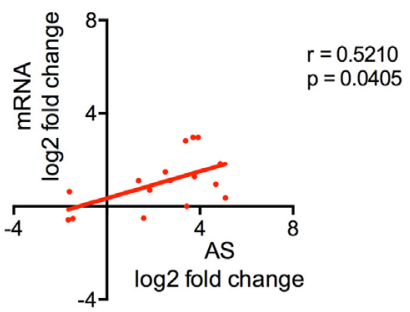

lincRNA

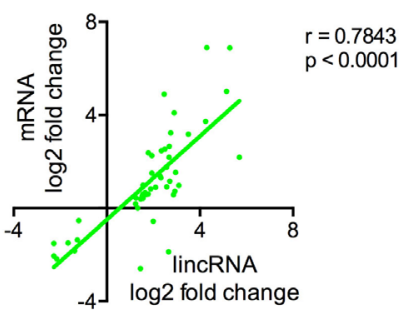

Antisense-Upstream

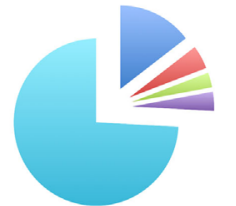

$\square$ SINE

- LINES

- LTR elements

- DNA elements

- Non-repeats

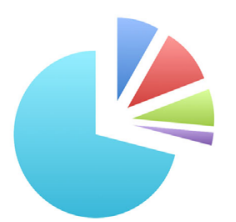

\section{Antisense-Downstream}

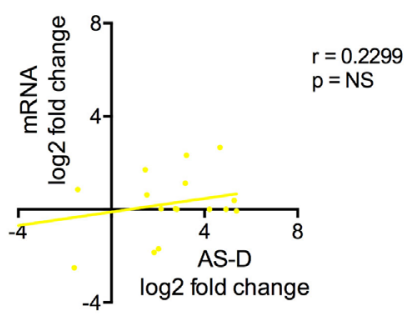

Antisense-Upstream

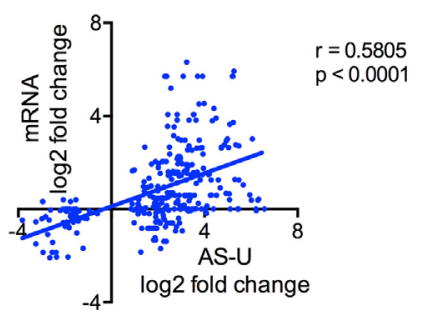


FIGURE 3 | Continued

Characterization of differentially expressed single exon long non-coding RNAs (IncRNAs) following activation of the human innate immune response. (A) Venn diagram showing the overlap in the single exon IncRNAs expression in resting monocytes, macrophages, epithelial cells, and chondrocytes. (B) Distribution of different IncRNA species in resting monocytes, macrophages, epithelial cells, and chondrocytes, and (C) Pie chart showing the percentage distribution of repeat sequences in the various sub-populations of IncRNAs across all four cell types with SINES = short interspersed nuclear elements, LINES = long interspersed nuclear elements, and LTR = long terminal repeat. (D) Pathways' analysis of the messenger RNAs (mRNAs) located within 1 Mb of the differentially expressed IncRNA. (E) Pearson's correlation between the differential expression of various IncRNA populations and that of the nearest mRNA.

Following ab initio assembly, we identified 2,386 lncRNAs across the four mouse cell types that could be divided into 869 lincRNAs (36\%), 1,201 AS (50\%), 170 AS-downstream (7\%), and 146 AS-upstream (6\%) (Table S8 in Supplementary Material). As a possible consequence of the poorer annotation of the mouse transcriptome, $1,592(67 \%)$ of these were found to be novel lncRNAs. Following LPS stimulation, we showed differential expression of 210 lncRNAs (133 lincRNA, 34 AS, 19 AS-downstream, and 24 AS-upstream; $q<0.05)$ including 69 in BMDMs, 158 in RAW macrophages, 7 in peritoneal macrophages, and 20 in dendritic cells (Figure 5C; Table S8 in Supplementary Material). As with human cells, examination of the overlap between cell types showed that the vast majority (171 or $81 \%$ ) were expressed in a cell-specific manner. Of the remainder, 36 were found in two cell types and 4 were found in three cell types (Figure 5C). Interestingly, our $a b$ initio assembly identified three lncRNAs that have previously been shown to regulate the innate immune response: lincRNA-COX2 (mXLOC_001674) (17, 18), lincRNA-EPS (mXLOC_029096) (19), and lincRNA-Tnfaip3 (mXLOC_003831) (20). Differential expression in response to LPS was seen with lincRNA-COX2 (BMDMs, RAW macrophages, and peritoneal macrophages) and lincRNA-EPS (RAW macrophages) but not lincRNA-Tnfaip3 (Table S9 in Supplementary Material).

Characterization of these differentially expressed lncRNAs showed that these were broadly similar to that observed in humans. Thus, these were found to be composed of $\sim 30 \%$ repeat elements (Figure 5D). Functional analysis identified 540 genes located within $1 \mathrm{Mb}$ of the differentially expressed lncRNAs and showed that these were associated with immune activation and response (Figure 5E), while the fold change in expression of the nearest mRNA was showed to correlate with the changes in AS $(r=0.7952, p<0.0001)$ and lincRNAs $(r=0.4209, p<0.0001)$ (Figure 5F).

Our analysis of sequencing data from multiple mouse cell types identified 210 lncRNAs that were differentially expressed following induction of the innate immune response. These demonstrated comparable characteristics to those observed in humans including cell-specific expression, large regions of repeat sequences, and correlation between their expression and that of local inflammatory genes.

\section{Identification of Microdomains in Differentially Expressed Human and Mouse IncRNAs}

It has been speculated that the action of lncRNAs is mediated through microdomains that interact with proteins or undergo base pairing with RNA and/or DNA. To identify potential microdomains, we searched for conserved sequences within our catalogs of differentially expressed lncRNA genes. As previously reported (46), our initial analysis of the evolutionary conservation of lncRNAs showed that these were poorly conserved (Figure 6A). Thus, using PhastCons (seven-way vertebrate), which determines conservation on a $0-1$ scale ( 1 being the most conserved), we obtained values of $0.162,0.165,0.151$, and 0.161 with the human AS, AS-downstream, AS-upstream, and lincRNAs, respectively. This value was significantly greater than the $0.099 \pm 0.002$ for the intronic regions of protein-coding genes ( $p<0.0001-$ Mann-Whitney $U$-test) but considerably less than the value for exonic, $5^{\prime}$ - and $3^{\prime}$-UTRs of protein-coding genes at $0.842 \pm 0.001,0.376 \pm 0.011$, and $0.373 \pm 0.001$, respectively (Figure 6A). Similarly, PhastCons analysis of the mouse catalog (vertebrate 60-way) produced values of 0.246 for AS and 0.182 for lincRNAs, which were significantly greater than those seen for intronic regions ( $p<0.0001-$ Mann-Whitney $U$-test) but less than the value for exonic, $5^{\prime}$ - and $3^{\prime}$-UTRs of protein-coding genes (Figure 6A).

Despite this poor overall evolutionary conservation, we proceeded to look for the presence of microdomains through comparison of the lncRNAs. This was performed following the removal of the repeat sequences using Repeatmasker (see text footnote 5). These lncRNAs gave a mean length of $4.7 \mathrm{~kb}$ (human) and $4.0 \mathrm{~kb}$ (mouse) and were shown to be rich in AT residues (58\% for human and 54\% for mouse). Analysis using MEME-ChIP (37) identified three conserved microdomains in the human lncRNA catalog but found nothing within the mouse lncRNA catalog (Figure 6B). In subsequent studies, we employed BLASTn (38) to identify shared regions within the human and mouse lncRNA catalogs. The output was compared with a comparable number of randomly generated control sequences and protein-coding genes, of similar lengths and AT composition. This analysis showed $<1 \%$ overall conservation but identified 5,130 and 4,199 significant hits of lengths 12-50 nt in the human and mouse lncRNA catalogs, respectively (Figure 6C). This was significantly higher $[p<0.0001$ : one-way analysis of variance (ANOVA)] than the 1,511 and 1,264 regions identified in the human and mouse control sequences. Protein-coding genes showed comparable number of hits to lncRNAs around the peak of $20 \mathrm{nt}$, but overall number of hits was elevated throughout the 12-50 nt range (Figure 6C). Submission of the BLASTn hits from the human and mouse lncRNA catalogs into MEME-ChIP identified 4 and 10 microdomains, respectively. No microdomains were detected in the controls. When we compared these motifs with the ATtRACT database of RNA-biding proteins and associated motifs (47), we found that a four of them (three in human and one in mouse) had positive hits with known RNA-binding proteins (Figures 6D,E). In general, these proteins were found to be involved in mRNA splicing, stability, and transport. Thus, despite 
A

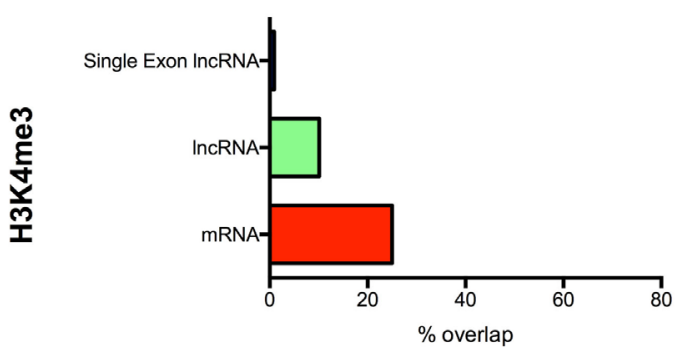

B

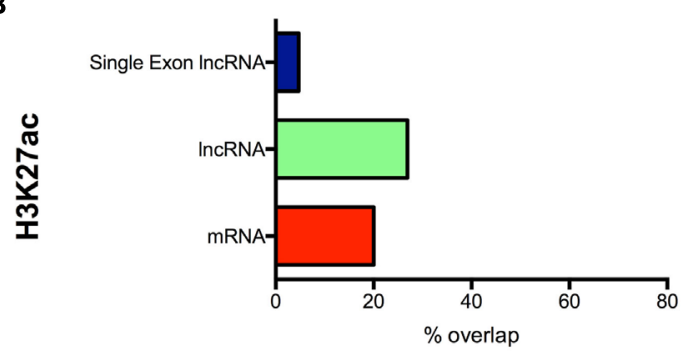

E
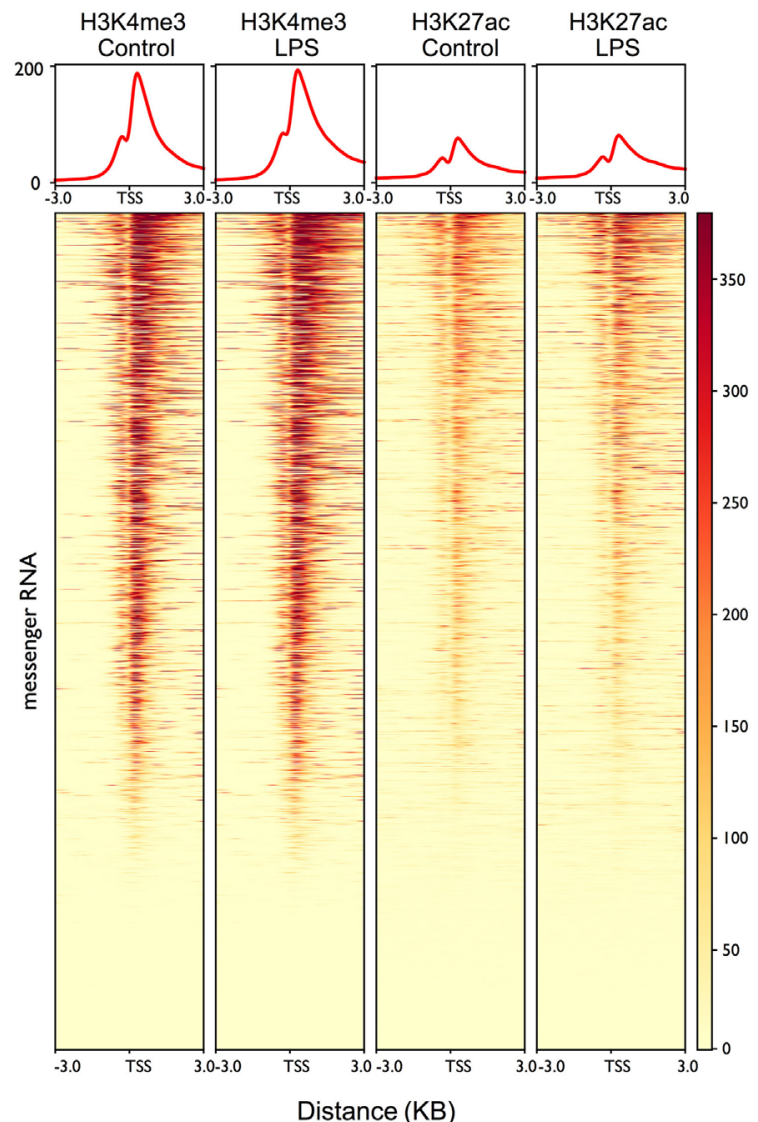

C LPS

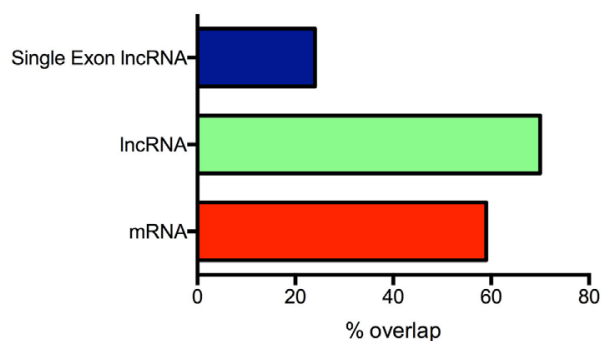

D

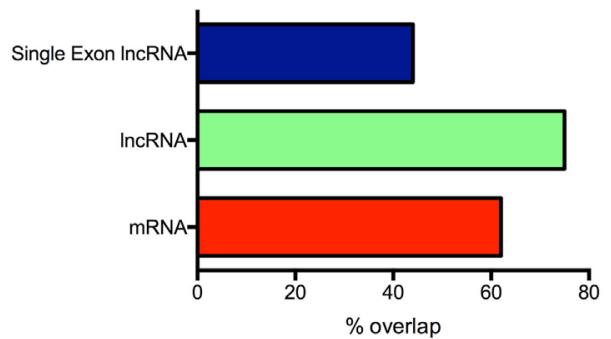

F - Single exon IncRNA

- Multi exon IncRNA

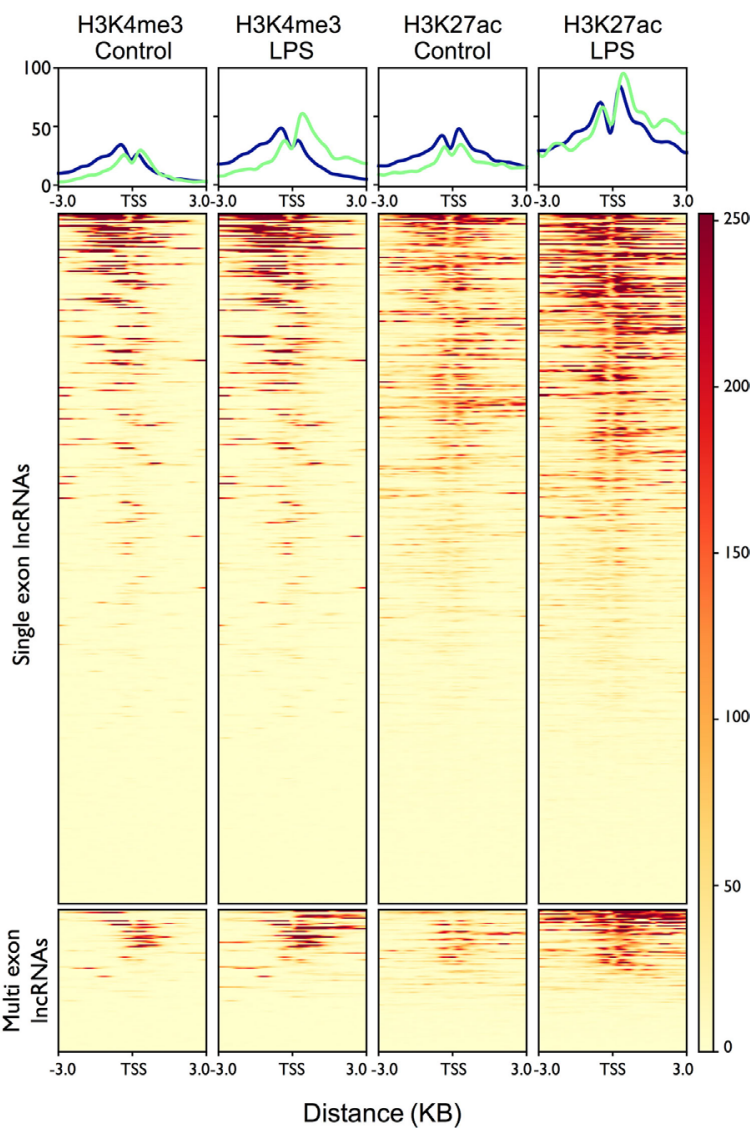

FIGURE 4 | Analysis of the overlap between mRNAs/IncRNAs and histone marks in human monocytes ChIPSeq data from resting (A,B) and lipopolysaccharides (LPS)-stimulated (C,D) is expressed as the percentage of long non-coding RNA (IncRNA) and messenger RNA (mRNA) genes that overlap with peaks identified by MACS2 (\% genomic overlap) for H3K4me3 (a marker of transcriptional activity) (A,C) and H3K27ac (a marker of active enhancers/promoters) (B,D). Heatmaps were generated (lower panel) displaying the deposition of H3K4me3 and H3K27ac across the promoters (TSS $\pm 3 \mathrm{~kb}$ ) of differentially expressed mRNAs (E) and IncRNAs (F). The upper panel displays the mean deposition of reads across all of the regions in the heatmap. 
A

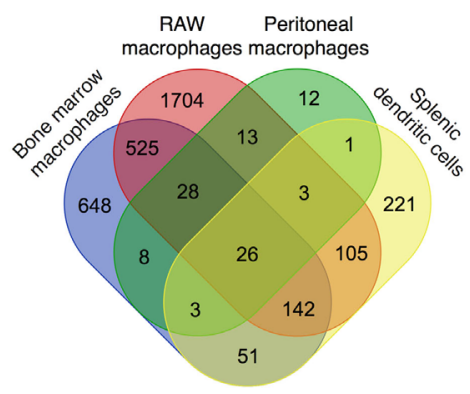

Differentially expressed protein coding genes

in mouse cells

B

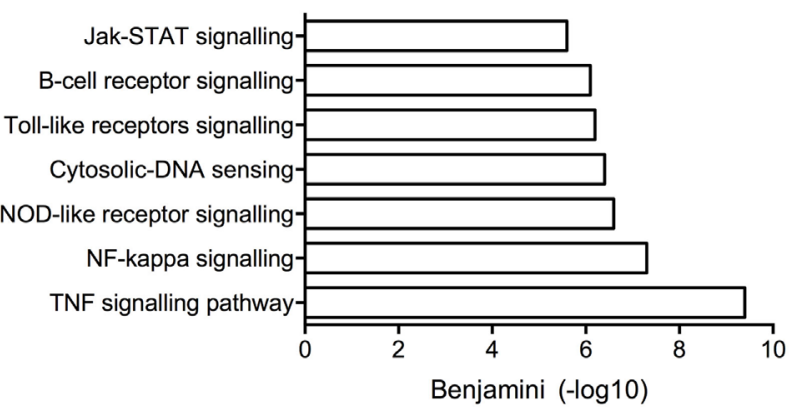

E

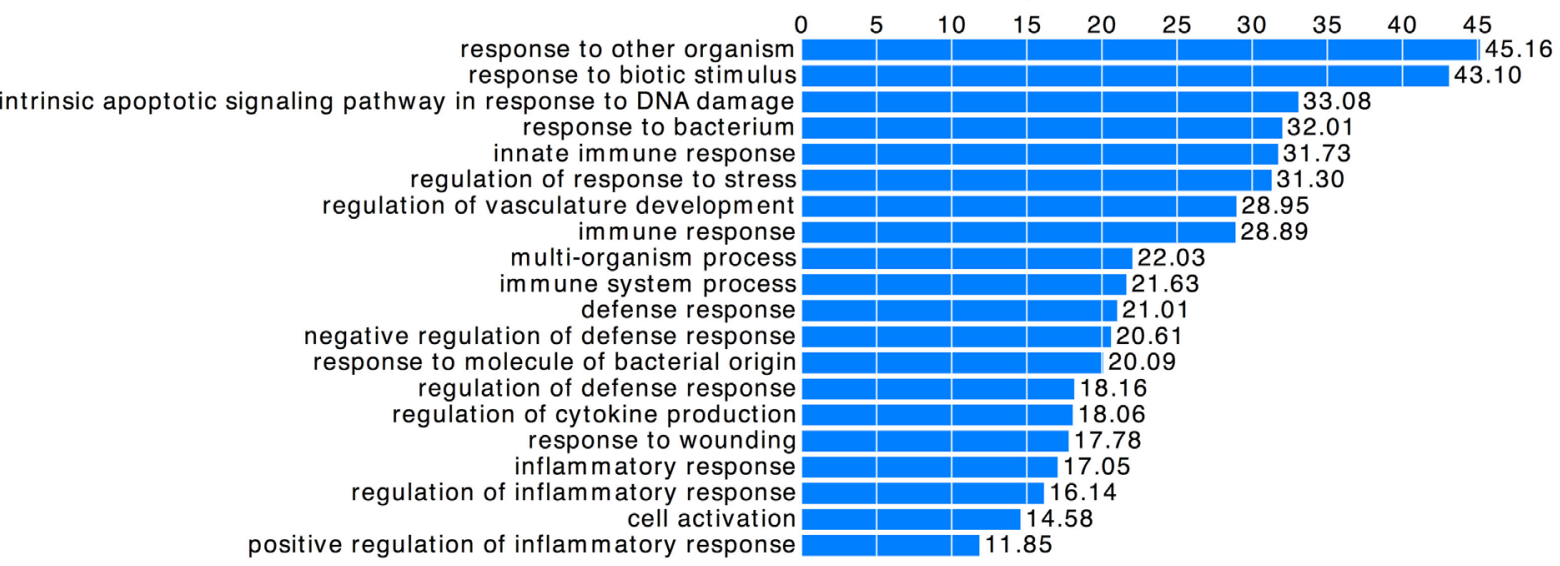

F

Antisense

lincRNA
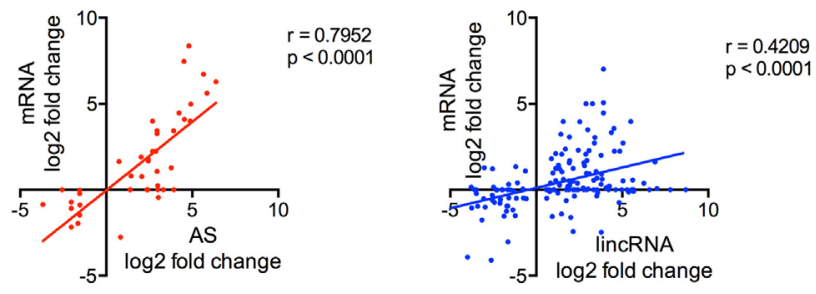

C

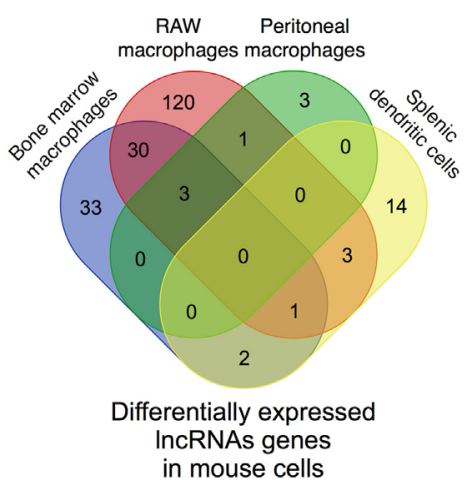

D SINES (Alu/MIR)

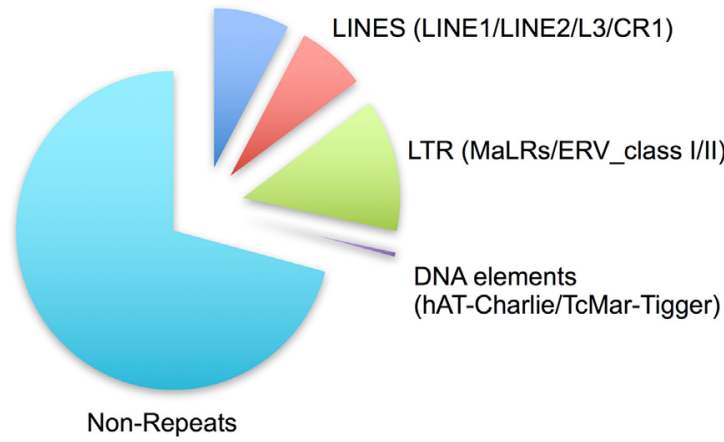

GO Biological Process

$-\log 10$ (Binomial $p$ value) response to other organism response to biotic stimulus

\begin{tabular}{|c|c|c|c|c|c|c|c|c|}
\hline \multicolumn{8}{|c|}{$-\log 10($ Binomial p value) } & \\
\hline 5 & 10 & 15 & 20 & 25 & 30 & 35 & 40 & 45 \\
\hline & & & & & & & 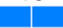 & 45.16 \\
\hline & & & & & & & & 43.10 \\
\hline & & & & & & 33.0 & & \\
\hline & & & & & & 2.01 & & \\
\hline & & & & & & 1.73 & & \\
\hline & & & & & & 1.30 & & \\
\hline & & & & & 28 & & & \\
\hline & & & & & 28. & & & \\
\hline & & & & 2.03 & & & & \\
\hline & & & & 1.63 & & & & \\
\hline & & & & .01 & & & & \\
\hline & & & & 61 & & & & \\
\hline & & & & & & & & \\
\hline & & & 18. & & & & & \\
\hline & & & 18. & & & & & \\
\hline & & & 17.7 & & & & & \\
\hline & & & 7.0 & & & & & \\
\hline & & & .14 & & & & & \\
\hline & & & & & & & & \\
\hline & & 1.8 & & & & & & \\
\hline
\end{tabular}


FIGURE 5 | Continued

Characterization of differentially expressed long non-coding RNAs (IncRNAs) following activation of the mouse innate immune response. (A) Venn diagram showing the overlap in the differentially expressed messenger RNAs (mRNAs) in lipopolysaccharides (LPS)-stimulated bone marrow macrophages, RAW 264.7 macrophages, peritoneal macrophages, and splenic dendritic cells. (B) Pathway analysis of the differentially expressed mRNA across all cell types. (C) Venn diagram showing the overlap in the differentially expressed IncRNAs in LPS-stimulated bone marrow macrophages, RAW 264.7 macrophages, peritoneal macrophages, and splenic dendritic cells. (D) Pie charts showing the percentage distribution of repeat sequences in the differentially expressed IncRNAs across all four cell types with SINES = short interspersed nuclear elements, LINES = long interspersed nuclear elements, and LTR = long terminal repeat. (E) Pathways analysis of the mRNAs located within $1 \mathrm{Mb}$ of the differentially expressed IncRNAs. (F) Pearson's correlation between the differential expression of various IncRNA populations and that of the nearest mRNA.

the poor evolutionary conservation, this analysis indicated that the differentially lncRNA contains short conserved regions or microdomains that might be important in mediating their functions and mechanism of action.

\section{Identification of Syntenic IncRNAs in the Human and Mouse Innate Immune Responses}

No homology was observed between the differentially expressed human and mouse lncRNA catalogs. Since it has been suggested that genomic position might be important to their biological action, we compared the human and mouse catalogs to identify those demonstrating synteny. Our analysis showed that $21(10 \%)$ of the differentially expressed human lncRNAs had syntenic versions in mice and included the two human lncRNAs that were differentially expressed in all human cell types, $h X L O C \_405581$ (which mapped to mouse mXLOC_025443) and hXLOC_367599 (which mapped to $m X L O C \_003168$ ) (Table S10 in Supplementary Material). However, only five of these syntenic mouselncRNAswere also significantly differentially expressed $(p<0.05)$ in a least one mouse cell type and included mXLOC_014053 (hXLOC_246791), mXLOC_039871 (hXLOC_039871), ENSMUSG00000097180 (hXLOC_376116), mXLOC_025443 (hXLOC_405581), and mXLOC_044198 (hXLOC_455493). With a mean of 9\%, BLASTn analysis showed increased conservation across these syntenic genes, compared to all differentially expressed lncRNAs $(<1 \%)$, although there was a wide variation (0-53\%) (Table S10 in Supplementary Material).

\section{IL7-AS Regulates the Inflammatory Response in Human and Mice}

In order to validate the sequencing data, we employed qRT-PCR to measure the levels of five lncRNAs across three human cell types (monocytes, macrophages, and epithelial A549 cells) and showed a significant correlation between the fold changes following differential expression (Figure 7A). To assess the biological relevance of these lncRNA catalogs, we examined the function of the syntenic lncRNAs, $h X L O C \_405581$ and mXLOC_025443, that is differentially expressed in multiple human and mouse cell types. These were renamed $h I L 7-A S$ and $m I L 7-A S$ as a result of their AS overlap with the promoter region of $I L 7$, a cytokine that has been implicated in T- and B-cell development (48). Analysis of the structure of human and mouse IL7-AS showed that these were complex genes that could be assembled potentially into multiple transcripts. In the case of the human hIL7-AS, sequencing data indicated the existence of up to nine exons (however, for simplicity, we have only shown the four most represented exons, Figure 7B), which could be assembled into potentially four transcripts of a gene of up to 10,280 nt in length (including 34\% repeat sequences). In contrast, the mouse mIL7-AS was somewhat less complex containing up to five exons that could be assembled into four potential transcripts giving a gene of an approximate length of 5,043 nt (including 63\% repeat sequences, Figure 7C).

To facilitate the functional analysis of $I L 7-A S$, experiments were performed using IL1 $\beta$-stimulated human A549 lung epithelial cells, LPS-stimulated human monocytic THP-1 cells, and LPS-stimulated mouse RAW 264.7 macrophages, which are amenable to transfection. In all cell types, measurement of time courses shows a similar rapid increase in hIL7-AS and mIL7-AS expressions, peaking between 4 and $6 \mathrm{~h}$ and remaining elevated at $24 \mathrm{~h}$ (Figure 7D). Examination of IL7 expression showed a parallel production of IL7 mRNA, albeit a smaller fold increase, in activated human A549 epithelium and THP-1 monocyte cells, but not mouse RAW 264.7 cells, where IL7 expression does not seem to be affected by LPS stimulation (Figure 7D). Although IL7 expression seems to correlate with the expression of hIL7-AS in A549 and THP-1 cells, it is interesting to note that the absolute expression of $I L 7$, according to our sequencing data, is at least 10 times lower than the expression of $h I L 7-A S$ (IL7 $=0.6$ FPKM vs $h I L 7-A S=7.0 \mathrm{FPKM}$ in A549 and $I L 7=5.5 \mathrm{FPKM}$ vs $h I L 7-$ $A S=182.2$ FPKM in monocyte).

Most lncRNAs present a bias toward nuclear localization, where previous studies of functional lncRNAs have been shown to regulate the transcription of protein-coding genes $(7,49,50)$. We therefore investigated the subcellular localization of IL7-AS in human cell lines (Figure 7E). Indeed, the expression of hIL7$A S$ was enriched in the nuclear fraction, compared to the whole lysate. We also looked at the expression of NEAT-1, a lncRNA known to be mainly located in the nucleus (51) and the mitochondrially encoded cytochrome B $(M T-C Y B)$ gene, produced from mitochondrial DNA in the cytoplasm (52). As expected, both NEAT-1 and MT-CYB were shown to be enriched, respectively, in the nucleus and in the cytoplasm fraction (Figure 7E), confirming that the separation procedure was successful.

To examine whether IL7-AS has a role in the innate immune response, we used AS locked nucleic acid (LNA) to knockdown the expression of IL7-AS RNA in both human and mouse. In human, we selected two AS LNAs targeting exon 1 (LNA 1) and exon 4 (LNA 2) that attenuated both IL1 $\beta$ - and LPS-induced hIL7-AS production by $50-85 \%$ at $24 \mathrm{~h}$ (Figure 7F) of A549 and THP-1 cells, respectively. Likewise, in mouse, selected AS LNAs, targeting exon 1 (LNA 1) and exon 3 (LNA 2), showed similar knockdown of $m I L 7-A S$ than in human cell lines (Figure 7F). Knockdown did not significantly impact upon $I L 7$ mRNA production indicating 
A

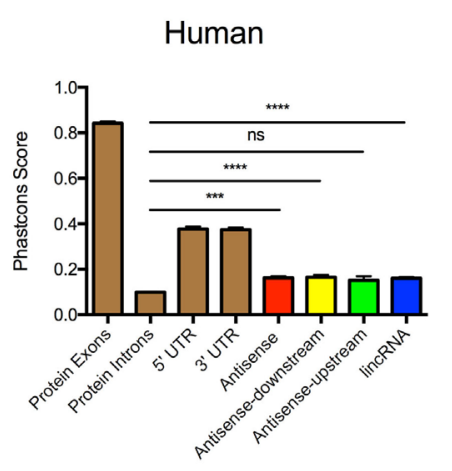

Mouse

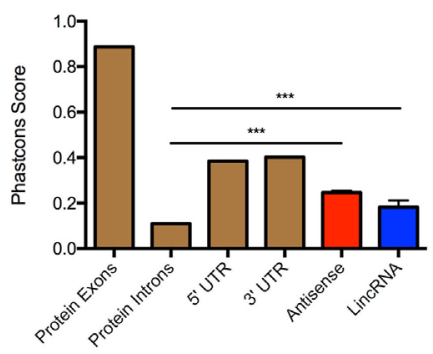

D

Human
B

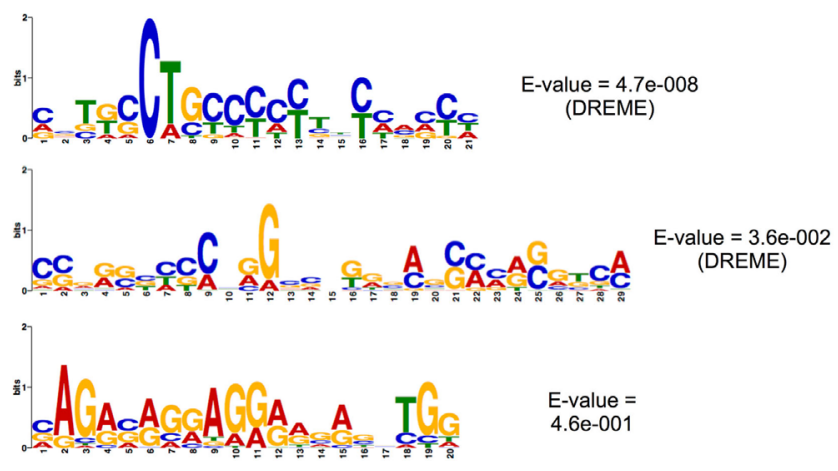

C
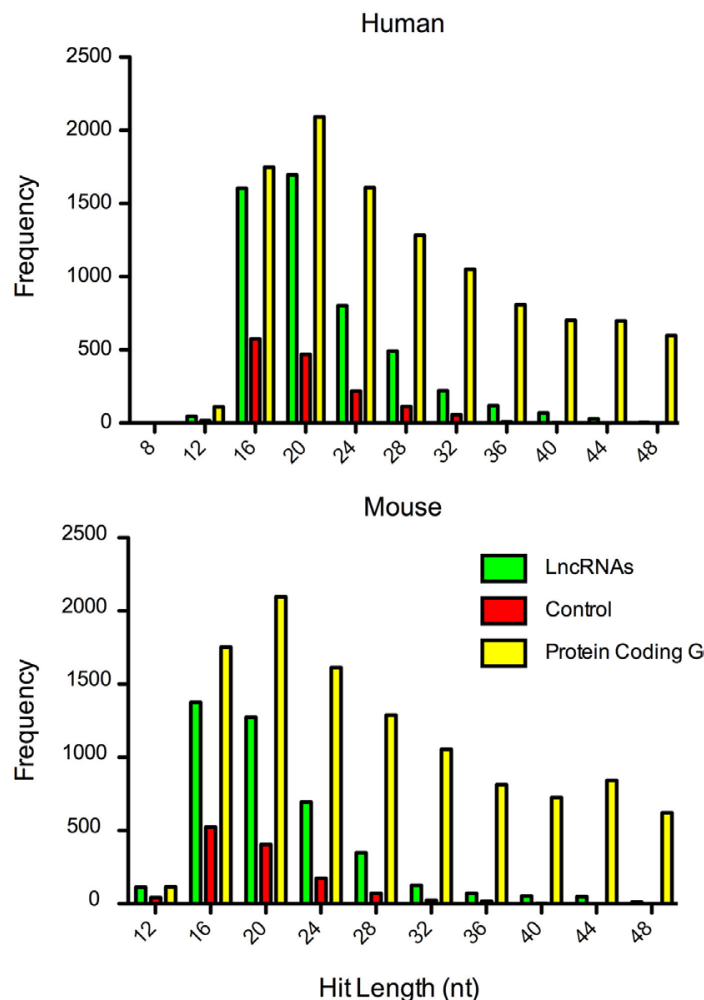

E
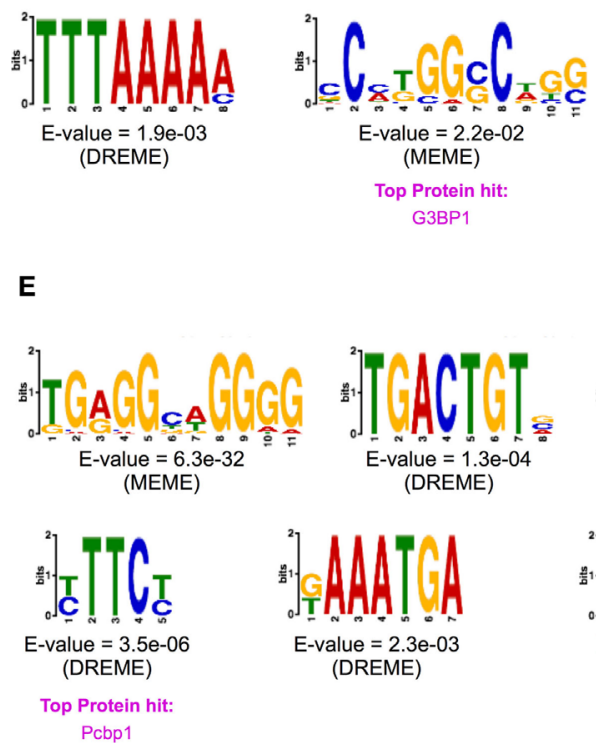
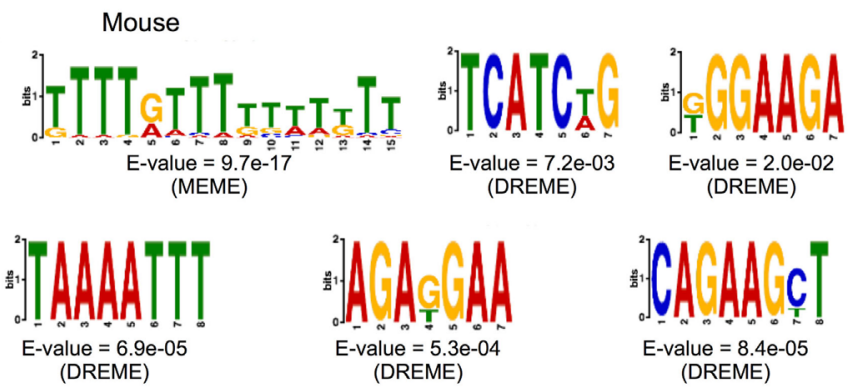

FIGURE 6 | Identification of conserved microdomains in the differentially expressed human and mouse long non-coding RNAs (IncRNAs). (A) PhastCons analysis of the conservation of the differentially expressed IncRNA species in human and mouse cells compared with the exon, intronic, and untranslated regions (UTRs) of protein-coding genes. (B) Identification of conserved microdomains within human IncRNA sequences using the MEME-suite (following removal of repeat sequences). (C) Distribution of hits obtained from the BLASTn analysis of IncRNAs, matched random controls, and matched protein-coding genes in human and mouse. (D,E) Identification of conserved microdomains within human (D) and mouse (E) BLASTn data. 

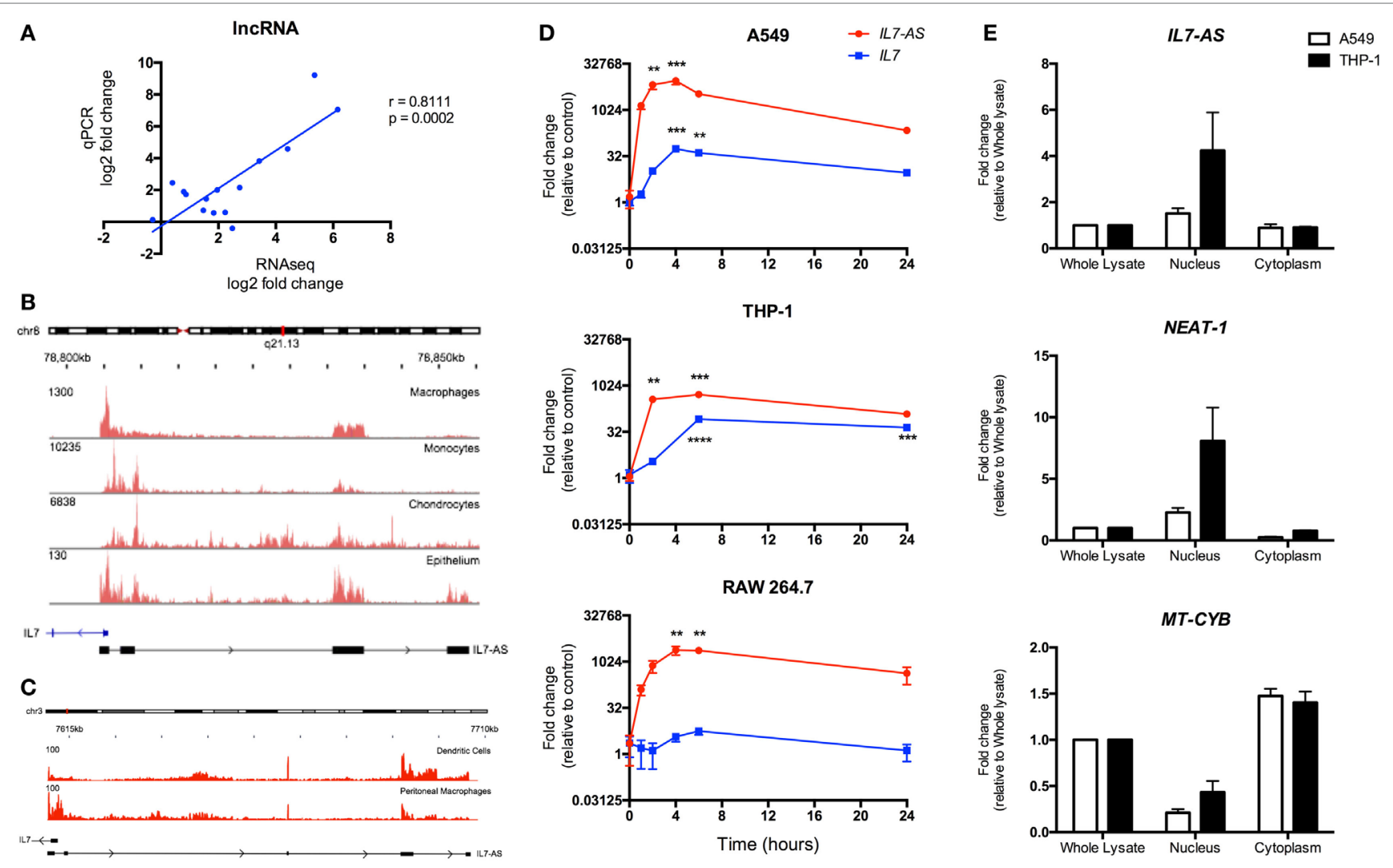

$\mathbf{F}$
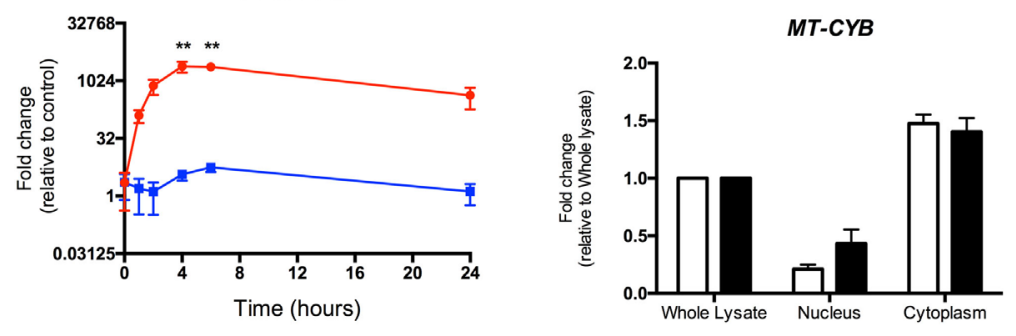

IL7-AS RNA

IL7 mRNA

IL6 mRNA

IL6 Cytokine
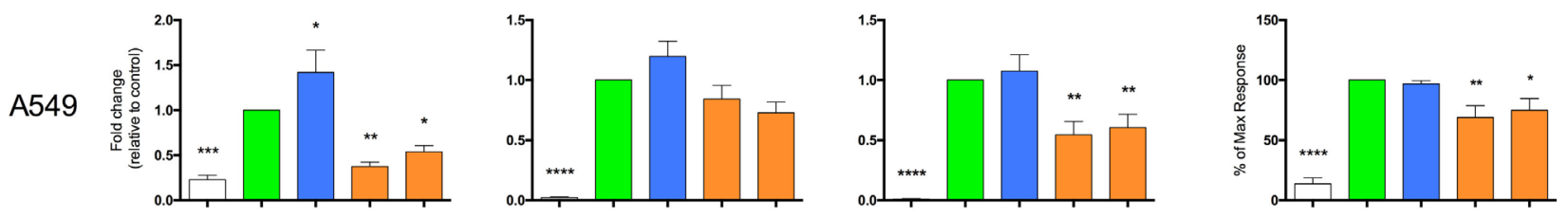

THP-1
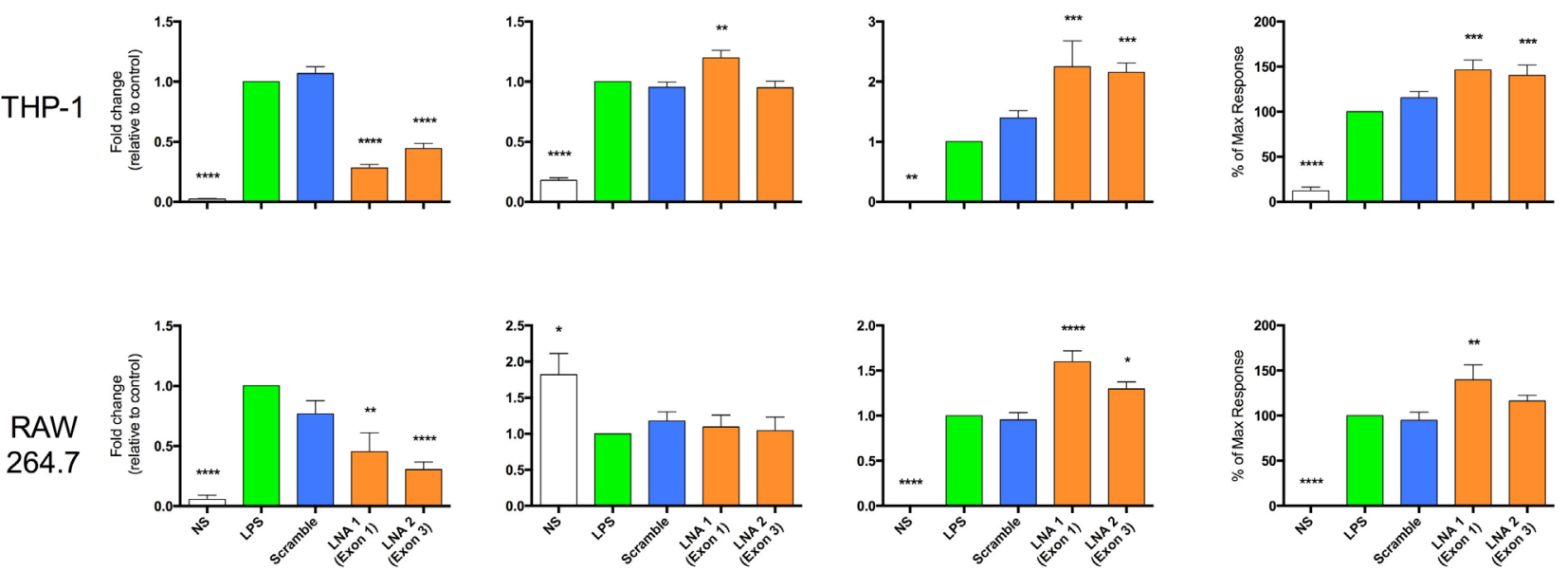

FIGURE 7 | Continued 


\section{FIGURE 7 | Continued}

IL7-AS regulates the expression and release of IL6 from interleukin-1 $\beta$ (IL1 $\beta$ )- and lipopolysaccharides (LPS)-stimulated human and mouse cells. (A) Comparison of fold change in expression of differentially expressed IncRNAs using RNA sequencing and qRT-PCR. Structure and profile of IL7-AS expression in (B) human and (C) mouse cells visualized using the Integrated Genomics Viewer (IGV). (D) Time course of IL7-AS and IL7 mRNA production in IL1 $\beta$-stimulated human alveolar A549 epithelium and LPS-stimulated human THP1 monocytes and mouse RAW 264.7 macrophages ( $n=3$ independent experiments). (E) Subcellular distribution of IL7-AS in IL1 $\beta$-stimulated A549 epithelium and LPS-stimulated THP1 monocytes in which NEAT-1 and mitochondrial-cytochrome b (MT-CYB) are employed as markers of nuclear and cytoplasmic fractions, respectively ( $n=4$ independent experiments). (F) Effect of transfection with a negative control LNA (scramble) or two antisense LNA (LNA 1 or 2) targeting, respectively, exons 1 and 4 (human cells) or exons 1 and 3 (mouse cells) of IL7-AS at a final concentration of 30 nM. Cells were then treated with either IL $1 \beta(30 \mathrm{ng} / \mathrm{ml})$ or LPS $(1 \mu \mathrm{g} / \mathrm{ml})$, or left untreated for $24 \mathrm{~h}$, prior to measurement of levels of the stated gene (by q-PCR) or proteins (by ELISA) ( $n=7-8$ independent experiments). Statistical significance was performed using either two-way analysis of variance (ANOVA) for time courses or repeated measure one-way ANOVA with both a Dunnett's post-test correction, where ${ }^{*} p<0.05,{ }^{* *} p<0.01,{ }^{\star \star *} p<0.001$, and ${ }^{* \star * *} p<0.0001$ versus control.

that although IL7-AS and IL7 overlap at their promoter region, the action of $I L 7-A S$ is not mediated through $I L 7$ regulation in cis (Figure 7F). Instead, these results suggest that any potential biological actions of IL7-AS might be mediated in trans. Indeed, IL7-AS knockdown showed significant modulation of the IL1 $\beta$ and LPS-induced expressions of the pro-inflammatory mediator IL6 on both mRNA production and release of the cytokine in human and in mouse (Figure 7F). Interestingly, IL1 $\beta$-induced IL6 production was significantly down-regulated in A549 cells upon IL7-AS knockdown, while in THP-1 and RAW 264.7 cells, knockdown showed an upregulation of LPS-induced IL6 production (Figure 7F). These results suggest that $I L 7-A S$ function on IL6 production is cell and/or stimuli specific.

Overall, these studies provide evidence of the utility of using differential expression as the basis for identifying functional lncRNA in the innate immune response and have for the first time identified a lncRNA (i.e., $I L 7-A S$ ) that regulates the inflammatory response in both human and mouse models.

\section{DISCUSSION}

Using next-generation sequencing data from four human and four mouse cell types, we have undertaken the first comprehensive analysis of the changes in lncRNA expression associated with the activation of the innate immune response. This is important since differential expression has commonly provided the initial step in the search for functional lncRNAs and has led to the identification a number that regulate the associated inflammatory response including PACER (13), THRIL (14), lncIL7R (15), and IL1 $\beta$-RBT46 (16) in humans and lincRNA-COX2 (17, 18), lincRNA-EPS (19), and lincRNA-Tnfaip3 (20) in mice. Differential expression has also been employed to compare T- and B-cell populations and identified lncRNAs that regulate multiple aspects of the adaptive immune response including activation, proliferation, and differentiation (53-55). Using this approach, we have demonstrated differential expression of 204 human and 210 mouse lncRNAs, which included PACER (13), IL1 $\beta$-RBT46 (16), lincRNA-COX2 (17, 18), and lincRNA-EPS (19). Intriguingly, we were unable to detect the expression of THRIL (14), $\operatorname{lnc}-I L 7 R$ (15), or lincRNA-Tnfaip3 (20), which were all located within the 3 ' UTRs of known protein-coding genes and were initially detected using microarrays. In future, we therefore believe that visual annotation of sequencing data should be the method of choice when identifying novel lncRNAs. In order to produce as comprehensive a lncRNA catalog as possible, sequencing of two of the eight cell types (human epithelial A549 cells and the mouse macrophage RAW264.7 cells) was undertaken after polyA+ selection (rather than ribozero selection). This is likely to influence the comparison between cell types and, specifically, might reduce the number of lncRNAs detected, since we would be unable to identify those lacking polyA+ tails. Although it is difficult to assess the potential impact of polyA+ versus ribozero isolation, the similarity in the numbers of differentially expressed lncRNAs in epithelial A549 cells (39 lncRNAs), compared with monocytes (105 lncRNAs), macrophages (50 lncRNAs), and chondrocytes ( 65 lncRNAs), indicates that we may be omitting only a small number.

As a part of our analysis, we also investigated the changes in single exon transcripts, which are traditionally excluded from the lncRNA classification (which requires two or more exons). This work suggests that, although a number of these are likely to regulate the innate immune response (i.e., PACER) (13), the large numbers and the wide variation between cell types indicate that the majority represent transcriptional noise and/or are an artifact of the analysis pathway. This conclusion is supported by the ChIPseq analysis in human monocytes that showed a poor overlap between markers of active transcription and enhancers/ promoters.

Analysis of the full-length genes demonstrated weak conservation through evolution, as well as between the lncRNAs $(<1 \%)$ and showed no homology between the human and mouse catalogs. However, a combination of BLASTn and MEME-ChIP allowed the identification of multiple conserved microdomains of lengths 5-30 nt. We speculate that these microdomains might mediate the actions of lncRNAs, either through protein binding and/or base pairing to RNA/DNA. Previous reports have uncovered only lncRNA-protein interactions, including an action of lincRNA-COX2 (17, 18), THRIL (14), and lincRNAEPS (19) through heterogeneous ribonucleoproteins and PACER via the p50 component of NF- $\mathrm{KB}$ (13). Of relevance, the identification of microdomains was performed following the removal of the repeats that comprised $\sim 30 \%$ of lncRNA sequences. However, it is possible that repeats are important in mediating the action of lncRNA, with previous studies showing that Alu repeats can activate the inflammasome (51) and contain binding sites for transcription factors involved in regulating the macrophage response to Mycobacterium tuberculosis infection (56).

It has also been suggested that the maintenance of genomic position relative to protein-coding genes (synteny) might be 
important in determining the lncRNA function. Comparison across humans and mouse identified 22 syntenic lncRNAs, of which five were differentially expressed in both species. This included IL7-AS (located AS to IL7), which was induced across multiple human and mouse cell types and demonstrated the largest changes in absolute expression among the syntenic lncRNAs. Measurement of IL6 transcription and secretion showed that IL7-AS was a positive regulator of IL1 $\beta$-induced inflammatory response in human A549 epithelial cell but a negative regulator in LPS-stimulated human THP-1 monocytes and mouse RAW 264.7 macrophages. Given our previous report showing that IL7-AS (or CILinc02) was a negative regulator of IL1 $\beta$-stimulated IL6 release from human chondrocytes (57), this indicates that its actions are cell-type specific rather than stimulus specific. Future studies will need to ascertain whether this is related to alternative splicing and/or cell-specific differences in lncRNA mechanisms. In addition, since IL7-AS is the first lncRNA to demonstrate function in the innate immune response in both human and mouse cell models, this provide an opportunity to compare the physiological role of lncRNAs across these two species.

In summary, we have for the first time cataloged and characterized those lncRNAs that are differentially expressed in multiple human and mouse cell types following activation of the innate immune response. However, further studies will be necessary to determine which other IncRNAs are functional from those two catalogs. Indeed, a refined list of functional lncRNAs could give us fewer and/or more defined microdomains. It is envisaged that this will provide an important resource for the discovery of functional lncRNAs and elucidation of their mechanism of action.

\section{AUTHOR CONTRIBUTIONS}

BR undertook the majority of laboratory based studies, assisted in the experimental design and analysis of data, and contributed to the writing of the manuscript. JH contributed toward the laboratory based studies, the bioinformatics analysis, and the writing of the paper. LD assisted with experimental design. SJ assisted with experimental design and contributed to the writing of the paper. ML conceived of the experimental design, undertook the majority of the analysis of data, and contributed to the writing of the manuscript.

\section{REFERENCES}

1. Djebali S, Davis CA, Merkel A, Dobin A, Lassmann T, Mortazavi A, et al. Landscape of transcription in human cells. Nature (2012) 489:101-8. doi:10.1038/nature11233

2. Doolittle WF. Is junk DNA bunk? A critique of ENCODE. Proc Natl Acad Sci US A (2013) 110:5294-300. doi:10.1073/pnas.1221376110

3. Nawrocki EP, Burge SW, Bateman A, Daub J, Eberhardt RY, Eddy SR, et al. Rfam 12.0: updates to the RNA families database. Nucleic Acids Res (2015) 43:D130-7. doi:10.1093/nar/gku1063

4. Morris KV, Mattick JS. The rise of regulatory RNA. Nat Rev Genet (2014) 15:423-37. doi:10.1038/nrg3722

5. Kozomara A, Griffiths-Jones S. miRBase: annotating high confidence microRNAs using deep sequencing data. Nucleic Acids Res (2013) 42:D68-73. doi:10.1093/nar/gkt1181

6. Derrien T, Johnson R, Bussotti G, Tanzer A, Djebali S, Tilgner H, et al. The GENCODE v7 catalog of human long noncoding RNAs: analysis of their

\section{FUNDING}

BR was supported by BBSRC grant (BB/K006223/1) and LD. This study was supported by the NIHR Respiratory Disease Biomedical Research Unit at the Royal Brompton and Harefield NHS Foundation Trust and Imperial College London.

\section{SUPPLEMENTARY MATERIAL}

The Supplementary Material for this article can be found online at http://journal.frontiersin.org/article/10.3389/fimmu.2017.01038/ full\#supplementary-material.

DATA SHEET S1 | Principle component analysis and hierarchical clustering. Analysis of the distribution of samples using principle component analysis and hierarchical clustering in monocytes, macrophages, epithelium, and chondrocytes.

DATA SHEET S2 | Expression of antisense, lincRNA, and antisense-upstream/ downstream are not correlated with the nearest protein-coding gene. The correlation (Spearman's correlation) between the expression of antisense (A), antisense upstream and downstream (B), and lincRNAs (C), demonstrating an FPKM > 1 in non-stimulated cells was examined using data from all four cell types (monocytes, macrophages, epithelium, and chondrocytes).

DATA SHEET S3 | Expression of THRIL in human monocytes and macrophages. Profile of forward (red) and reverse (blue) sequence reads in control and LPSstimulated human monocytes and macrophages indicates the presence of BRI3BP but not the THRIL.

TABLE S1 | LNA antisense sequence and qRT-PCR probes.

TABLE S2 | Bioinformatics software and websites.

TABLE S3 | Gencode v23 protein-coding genes across four human cell types.

TABLE S4 | Long non-coding RNA expression across four human cell types.

TABLE S5 | Differentially expressed long non-coding RNA expression across four human cell types.

TABLE S6 | Differentially expressed single exon long non-coding RNA expression across four human cell types.

TABLE S7 | Mouse Gencode m12 gene expression across four mouse cell types.

TABLE S8 | Long non-coding RNA expression across four mouse cell types.

TABLE s9 | Differentially expressed long non-coding RNA expression across four mouse cell types.

TABLE S10 | Syntenic long non-coding RNAs between humans and mouse.

gene structure, evolution, and expression. Genome Res (2012) 22:1775-89. doi:10.1101/gr.132159.111

7. Ulitsky I, Bartel DP. lincRNAs: genomics, evolution, and mechanisms. Cell (2013) 154:26-46. doi:10.1016/j.cell.2013.06.020

8. Mattick JS, Rinn JL. Discovery and annotation of long noncoding RNAs. Nat Struct Mol Biol (2015) 22:5-7. doi:10.1038/nsmb.2942

9. Guttman M, Rinn JL. Modular regulatory principles of large non-coding RNAs. Nature (2012) 482:339-46. doi:10.1038/nature10887

10. Necsulea A, Soumillon M, Warnefors M, Liechti A, Daish T, Zeller U, et al. The evolution of lncRNA repertoires and expression patterns in tetrapods. Nature (2014) 505:635-40. doi:10.1038/nature12943

11. Brubaker SW, Bonham KS, Zanoni I, Kagan JC. Innate immune pattern recognition: a cell biological perspective. Annu Rev Immunol (2015) 33:257-90. doi:10.1146/annurev-immunol-032414-112240

12. Hennessy EJ, Parker AE, O’Neill LA. Targeting toll-like receptors: emerging therapeutics? Nat Rev Drug Discov (2010) 9:293-307. doi:10.1038/ nrd3203 
13. Krawczyk M, Emerson BM. p50-associated COX-2 extragenic RNA (PACER) activates COX-2 gene expression by occluding repressive NF-кB complexes. Elife (2014) 3:e01776. doi:10.7554/eLife.01776

14. Li Z, Chao T-C, Chang K-Y, Lin N, Patil VS, Shimizu C, et al. The long noncoding RNA THRIL regulates TNF $\alpha$ expression through its interaction with hnRNPL. Proc Natl Acad Sci U S A (2013) 111:1002-7. doi:10.1073/ pnas. 1313768111

15. Cui H, Xie N, Tan Z, Banerjee S, Thannickal VJ, Abraham E, et al. The human long noncoding RNA, lnc-IL7R, regulates inflammatory response. Eur J Immunol (2014) 44:2085-95. doi:10.1002/eji.201344126

16. IIott NE, Heward JA, Roux BT, Tsitsiou E, Fenwick PS, Lenzi L, et al. Long non-coding RNAs and enhancer RNAs regulate the lipopolysaccharideinduced inflammatory response in human monocytes. Nat Commun (2014) 5:3979. doi: $10.1038 /$ ncomms 4979

17. Guttman M, Amit I, Garber M, French C, Lin MF, Feldser D, et al. Chromatin signature reveals over a thousand highly conserved large non-coding RNAs in mammals. Nature (2009) 458:223-7. doi:10.1038/nature07672

18. Carpenter S, Aiello D, Atianand MK, Ricci EP, Gandhi P, Hall LL, et al. A long noncoding RNA mediates both activation and repression of immune response genes. Science (2013) 341:789-92. doi:10.1126/science.1240925

19. Atianand MK, Hu W, Satpathy AT, Shen Y, Ricci EP, Alvarez-Dominguez JR, et al. A long noncoding RNA lincRNA-EPS acts as a transcriptional brake to restrain inflammation. Cell (2016) 165:1672-85. doi:10.1016/j.cell.2016.05.075

20. Ma S, Ming Z, Gong A-Y, Wang Y, Chen X, Hu G, et al. A long noncoding RNA, lincRNA-Tnfaip3, acts as a coregulator of NF- $\mathrm{KB}$ to modulate inflammatory gene transcription in mouse macrophages. FASEB J (2017) 31:1215-25. doi:10.1096/fi.201601056R

21. Hutchins AP, Takahashi Y, Miranda-Saavedra D. Genomic analysis of LPSstimulated myeloid cells identifies a common pro-inflammatory response but divergent IL-10 anti-inflammatory responses. Sci Rep (2015) 5:885. doi:10.1038/srep09100

22. Kim D, Pertea G, Trapnell C, Pimentel H, Kelley R, Salzberg SL. TopHat2: accurate alignment of transcriptomes in the presence of insertions, deletions and gene fusions. Genome Biol (2013) 14:R36. doi:10.1186/gb-2013-14-4-r36

23. Kim D, Langmead B, Salzberg SL. HISAT: a fast spliced aligner with low memory requirements. Nat Methods (2015) 12:357-60. doi:10.1038/ nmeth. 3317

24. Li H, Handsaker B, Wysoker A, Fennell T, Ruan J, Homer N, et al. The sequence alignment/map format and SAMtools. Bioinformatics (2009) 25:2078-9. doi:10.1093/bioinformatics/btp352

25. Barnett DW, Garrison EK, Quinlan AR, Stromberg MP, Marth GT. BamTools: a C++ API and toolkit for analyzing and managing BAM files. Bioinformatics (2011) 27:1691-2. doi:10.1093/bioinformatics/btr174

26. Pertea M, Pertea GM, Antonescu CM, Chang T-C, Mendell JT, Salzberg SL. StringTie enables improved reconstruction of a transcriptome from RNA-seq reads. Nat Biotechnol (2015) 33:290-5. doi:10.1038/nbt.3122

27. Pertea M, Kim D, Pertea GM, Leek JT, Salzberg SL. Transcript-level expression analysis of RNA-seq experiments with HISAT, StringTie and Ballgown. Nat Protoc (2016) 11:1650-67. doi:10.1038/nprot.2016.095

28. Trapnell C, Williams BA, Pertea G, Mortazavi A, Kwan G, van Baren MJ, et al. Transcript assembly and quantification by RNA-Seq reveals unannotated transcripts and isoform switching during cell differentiation. Nat Biotechnol (2010) 28:511-5. doi:10.1038/nbt.1621

29. Harrow J, Frankish A, Gonzalez JM, Tapanari E, Diekhans M, Kokocinski F, et al. GENCODE: the reference human genome annotation for The ENCODE Project. Genome Res (2012) 22:1760-74. doi:10.1101/ gr.135350.111

30. Quinlan AR, Hall IM. BEDTools: a flexible suite of utilities for comparing genomicfeatures. Bioinformatics (2010) 26:841-2. doi:10.1093/bioinformatics/ btq033

31. Kong L, Zhang Y, Ye ZQ, Liu XQ, Zhao SQ, Wei L, et al. CPC: assess the protein-coding potential of transcripts using sequence features and support vector machine. Nucleic Acids Res (2007) 35:W345-9. doi:10.1093/nar/ gkm391

32. Mudge JM, Harrow J. Creating reference gene annotation for the mouse C57BL6/J genome assembly. Mamm Genome (2015) 26:366-78. doi:10.1007/ s00335-015-9583-x

33. Sturn A, Quackenbush J, Trajanoski Z. Genesis: cluster analysis of microarray data. Bioinformatics (2002) 18:207-8. doi:10.1093/bioinformatics/18.1.207
34. Ramskold D, Wang ET, Burge CB, Sandberg R. An abundance of ubiquitously expressed genes revealed by tissue transcriptome sequence data. PLoS Comput Biol (2009) 5:e1000598. doi:10.1371/journal.pcbi. 1000598

35. Afgan E, Baker D, van den Beek M, Blankenberg D, Bouvier D, Čech M, et al. The Galaxy platform for accessible, reproducible and collaborative biomedical analyses: 2016 update. Nucleic Acids Res (2016) 44:W3-10. doi:10.1093/ nar/gkw343

36. Kent WJ, Sugnet CW, Furey TS, Roskin KM, Pringle TH, Zahler AM, et al. The human genome browser at UCSC. Genome Res (2002) 12:996-1006. doi:10.1101/gr.229102

37. Bailey TL, Boden M, Buske FA, Frith M, Grant CE, Clementi L, et al. MEME SUITE: tools for motif discovery and searching. Nucleic Acids Res (2009) 37:W202-8. doi:10.1093/nar/gkp335

38. Camacho C, Coulouris G, Avagyan V, Ma N, Papadopoulos J, Bealer K, et al. BLAST+: architecture and applications. BMC Bioinformatics (2009) 10:421. doi:10.1186/1471-2105-10-421

39. Medina-Rivera A, Defrance M, Sand O, Herrmann C, Castro-Mondragon JA, Delerce J, et al. RSAT 2015: regulatory sequence analysis tools. Nucleic Acids Res (2015) 43:W50-6. doi:10.1093/nar/gkv362

40. Novakovic B, Habibi E, Wang S-Y, Arts RJW, Davar R, Megchelenbrink W, et al. $\beta$-Glucan reverses the epigenetic state of LPS-induced immunological tolerance. Cell (2016) 167:1354-68.e14. doi:10.1016/j.cell.2016. 09.034

41. Langmead B, Salzberg SL. Fast gapped-read alignment with Bowtie 2. Nat Methods (2012) 9:357-9. doi:10.1038/nmeth.1923

42. Ramírez F, Ryan DP, Grüning B, Bhardwaj V, Kilpert F, Richter AS, et al. deepTools2: a next generation web server for deep-sequencing data analysis. Nucleic Acids Res (2016) 44:W160-5. doi:10.1093/nar/gkw257

43. Zhang Y, Liu T, Meyer CA, Eeckhoute J, Johnson DS, Bernstein BE, et al. Model-based analysis of ChIP-Seq (MACS). Genome Biol (2008) 9:R137. doi:10.1186/gb-2008-9-9-r137

44. Huang DW, Sherman BT, Lempicki RA. Bioinformatics enrichment tools: paths toward the comprehensive functional analysis of large gene lists. Nucleic Acids Res (2009) 37:1-13. doi:10.1093/nar/gkn923

45. Johnson R, Guigo R. The RIDL hypothesis: transposable elements as functional domains of long noncoding RNAs. RNA (2014) 20:959-76. doi:10.1261/ rna.044560.114

46. Kutter C, Watt S, Stefflova K, Wilson MD, Goncalves A, Ponting CP, et al. Rapid turnover of long noncoding RNAs and the evolution of gene expression. PLoS Genet (2012) 8:e1002841. doi:10.1371/journal.pgen.1002841

47. Giudice G, Sánchez-Cabo F, Torroja C, Lara-Pezzi E. ATtRACT-a database of RNA-binding proteins and associated motifs. Database (Oxford) (2016) 2016:baw035. doi:10.1093/database/baw035

48. Niu N, Qin X. New insights into IL-7 signaling pathways during early and late T cell development. Cell Mol Immunol (2013) 10:187-9. doi:10.1038/ cmi.2013.11

49. Rinn JL, Chang HY. Genome regulation by long noncoding RNAs. Annu Rev Biochem (2012) 81:145-66. doi:10.1146/annurev-biochem-051410-092902

50. Cabili MN, Dunagin MC, McClanahan PD, Biaesch A, PadovanMerhar O, Regev A, et al. Localization and abundance analysis of human lncRNAs at single-cell and single-molecule resolution. Genome Biol (2015) 16:20. doi:10.1186/s13059-015-0586-4

51. Hutchinson JN, Ensminger AW, Clemson CM, Lynch CR, Lawrence JB, Chess A. A screen for nuclear transcripts identifies two linked noncoding RNAs associated with SC35 splicing domains. BMC Genomics (2007) 8:39. doi:10.1186/1471-2164-8-39

52. Anderson S, Bankier AT, Barrell BG, Debruijn M, Coulson AR, Drouin J, et al. Sequence and organization of the human mitochondrial genome. Nature (1981) 290:457-65. doi:10.1038/290457a0

53. Hu G, Tang Q, Sharma S, Yu F, Escobar TM, Muljo SA, et al. Expression and regulation of intergenic long noncoding RNAs during $\mathrm{T}$ cell development and differentiation. Nat Immunol (2013) 14:1190-8. doi:10.1038/ni.2712

54. Ranzani V, Rossetti G, Panzeri I, Arrigoni A, Bonnal RJP, Curti S, et al. The long intergenic noncoding RNA landscape of human lymphocytes highlights the regulation of T cell differentiation by linc-MAF-4. Nat Immunol (2015) 16:318-25. doi:10.1038/ni.3093

55. Casero D, Sandoval S, Seet CS, Scholes J, Zhu Y, Ha VL, et al. Long non-coding RNA profiling of human lymphoid progenitor cells reveals transcriptional 
divergence of B cell and T cell lineages. Nat Immunol (2015) 16:1282-91. doi:10.1038/ni.3299

56. Bouttier M, Laperriere D, Memari B, Mangiapane J, Fiore A, Mitchell E, et al. Alu repeats as transcriptional regulatory platforms in macrophage responses to M. tuberculosis infection. Nucleic Acids Res (2016) 44:10571-87. doi:10.1093/nar/gkw782

57. Pearson MJ, Philp AM, Heward JA, Roux BT, Walsh DA, Davis ET, et al. Long intergenic noncoding RNAs mediate the human chondrocyte inflammatory response and are differentially expressed in osteoarthritis cartilage. Arthritis Rheumatol (2016) 68:845-56. doi:10.1002/art.39520
Conflict of Interest Statement: The authors declare that the research was conducted in the absence of any commercial or financial relationships that could be construed as a potential conflict of interest.

Copyright $\odot 2017$ Roux, Heward, Donnelly, Jones and Lindsay. This is an open-access article distributed under the terms of the Creative Commons Attribution License (CC $B Y)$. The use, distribution or reproduction in other forums is permitted, provided the original author(s) or licensor are credited and that the original publication in this journal is cited, in accordance with accepted academic practice. No use, distribution or reproduction is permitted which does not comply with these terms. 